\title{
CABARET in the Ocean Gyres
}

S.A. Karabasov*1,4 ${ }^{1, \text { P.S. Berloff }}{ }^{2,3}$, and V.M. Goloviznin ${ }^{4}$

${ }^{1}$ University of Cambridge, Department of Engineering, Whittle Laboratory, Cambridge, UK

${ }^{2}$ Imperial College London, Grantham Institute for Climate Change and Department of Mathematics, London, UK

${ }^{3}$ Woods Hole Oceanographic Institution, Physical Oceanography Department, Woods Hole, USA ${ }^{4}$ Moscow Institute of Nuclear Safety of Russian Academy of Science, Moscow, Russia

* Corresponding author: 1 JJ Thompson Avenue, Cambridge, CB3 0DY, Tel: +44 1223 337599, Email: sak36@eng.cam.ac.uk 


\title{
CABARET in the Ocean Gyres
}

\author{
S.A. Karabasov, P.S. Berloff, and V.M. Goloviznin
}

\section{Abstract}

A new high-resolution Eulerian numerical method is proposed for modelling quasigeostrophic ocean dynamics in eddying regimes. The method is based on a novel, second-order non-dissipative and lowdispersive conservative advection scheme called CABARET. The properties of the new method are compared with those of several high-resolution Eulerian methods for linear advection and gas dynamics. Then, the CABARET method is applied to the classical model of the double-gyre ocean circulation and its performance is contrasted against that of the common vorticity-preserving Arakawa method. In turbulent regimes, the new method permits credible numerical simulations on much coarser computational grids.

Key words: mesoscale ocean dynamics, eddy resolving simulations, high-resolution schemes

\section{Introduction}

In many aspects mesoscale oceanic eddies, operating on the lengthscales of $\mathrm{O}(1-100) \mathrm{km}$ are analogous to the cyclones and anticyclones that constitute the atmospheric weather phenomenon. The problem of resolving these eddies in a dynamically consistent way is very important for ocean modelling and, therefore, for global climate predictions. For achieving high Reynolds number (Re) simulations, which are required for accurate modelling of the ocean, the models have to account for all important scales of motion. 
Modern ocean models enter a new phase in which eddies will be, at least, permitted in the numerical simulation. For such models advection scheme is a very important component. A crucial element of numerical advection scheme is its ability to propagate finite-amplitude and -phase disturbances on a discrete grid either without generating spurious short-wave oscillations, because of not preserving the correct dispersion relation i.e., dispersion error, or any considerable damping of the amplitude i.e., dissipation error (e.g., Kravchenko and Moin, 1997; Pope, 2000). Note, that the generation of short-wave oscillations is particularly detrimental in case an inverse energy cascade takes place, and the small scales affect large scales (e.g., Tabeling, 2002, Vallis, 2006). In the present paper the effect of spurious small-scale dispersion and dissipation on important large-scale properties of the solution are captured by comparing numerical predictions obtained with two different advection methods implemented within the same quasigeostrophic ocean modelling code (Berloff et al., 2007), which solves for the classical double-gyre problem (Holland, 1978). The original model implements standard eddy viscosity for parameterising effects of the unresolved scales of turbulent diffusion and conservative second-order Arakawa scheme for advection (Arakawa, 1966). In this paper, we consider a few versions of the original code based on different advection methods. Comparisons with the converged fine-grid solutions are made to investigate effects of numerical advection schemes on the coarse-grid solutions.

Solving 'convection-dominated' problems is a longstanding challenge for computational fluid dynamics (e.g., Rozhdestvensky and Yanenko, 1978; Roach, 1982; Hirsch, 2007). One of the difficulties is that the conventional second-order finite-difference schemes have large dispersion errors, which generate spurious ripples in the solutions. To counterbalance this effect, a numerical dissipation, such as the classical von Neumann and Richtmyer's (1950) artificial viscosity for compression-type pressure waves or such as the eddy viscosity in ocean circulation models, is added to the governing 
equations. However, a common negative side of this approach is the associated spurious dissipation that smears the large eddies along with the spurious ripples.

There are 3 general approaches for solving 'convection-dominated' problems: Eulerian, Lagrangian, and mixed Eulerian-Lagrangian. For the Eulerian methods, significant presence of both numerical dissipation and dispersion is common drawback. It is partially overcome in the Lagrangian and Eulerian-Lagrangian methods, which describe flow advection by following fluid particle coordinates, rather than by considering fixed coordinates on the Eulerian grid (e.g., Dritschel et al., 1999; Mohebalhojeh and Dritschel, 2004). A remarkable property of the Lagrangian methods is that they are exact for the linear-advection problem with a uniform velocity field, and in this case their accuracy is limited only by the accuracy of solving the corresponding Ordinary Differential Equations (ODEs), rather than by the accuracy of solving the full Partial Differential Equations (PDEs), as in the Eulerian case. The Lagrangian methods can be very efficient for simulations with multiple contact discontinuities and shocks, e.g., as those of multi-phase flows (e.g., Margolin and Shashkov, 2004). However, for the problems dominated by chaotic folding and stretching of the material lines, the Lagrangian methods have to be complemented with ad hoc 'repair' (or 'contour surgery') procedures. They allow to remove redundant Lagrangian particles that aggregate in some locations and to add new particles to where they are needed. The 'repair' procedure can be viewed as a special kind of numerical dissipation that drains energy from the underresolved scales. The numerical effect of the 'repair' procedure on the numerical dissipation and dispersion error always limits Re for the resolved scales. Also, specifying standard physical boundary conditions (e.g., no-slip, free-slip, etc.) is problematic for the Lagrangian methods.

The implementation of Eulerian-Lagrangian methods, including semi-Lagrangian and vortexin-a-cell methods is less intricate, since they employ an Eulerian interpolation/projection step after a Lagrangian step. However, they are no longer exact for the linear advection, and they are prone to the 
same dissipation and dispersion error problems as the Eulerian methods. For example, the mixed Eulerian-Lagrangian methods use artificial numerical dissipation for avoiding the 'repair' procedure and for ensuring a 'non-oscillatory' solution (e.g., Margolin, 1997). Finally, the Lagrangian-to-Eulerian grid interpolation is also a source of the numerical dissipation and dispersion errors.

Many Eulerian methods for 'convection-dominated' flows are based on emphasizing a particular property of the governing equations. Using such property as the basis for a "low-order" (firstor second-order) scheme, typically, further upgrades (e.g., high-order, non-oscillatory sequels, etc.) of the original scheme are developed. One such example is the family of optimized low-dispersion and low-dissipation finite-difference schemes, including implicit compact finite-difference schemes based on Pade-type approximations (Lele, 1992) and explicit schemes (Tam and Webb, 1993; Bogey and Bailly, 2004) that are popular in turbulence modelling. In particular, the family of explicit schemes was developed to overcome typical problems of the spectral and pseudo-spectral methods, such as handling non-periodic boundary conditions and suitability for parallel computations. The optimized explicit finite-difference schemes, typically, employ non-conservative forms of the governing equations, and use large computational stencils for more accurate approximation of the linear-wave dispersion relation in the physical domain. In order to cope with the large unresolved gradients emerging in nonlinear flows (e.g., shock waves) the high-order low-dissipative low-dispersive schemes use special filtering procedures (e.g., Robert, 1966; Asselin, 1972; Zhou and Wei, 2003). Such filtering is analogous to some form of artificial viscosity added to the scheme and is required, essentially, to reduce the order of the scheme in the vicinity of the unresolved solution gradients, in accordance with the Godunov Theorem (Godunov, 1959), that states that any monotone finite-difference scheme is first-order accurate.

Another example consists of the so-called "high-resolution schemes" (after A. Harten) that are based on Flux Corrected Transport (FCT), Total Variation Diminishing (TVD) solution ideas for quasi- 
linear hyperbolic conservation laws (e.g., Boris et al 1975; Harten et al, 1987; LeVeque, 2002). In this approach a conservative approximation of the governing equations is used, that has several useful properties such as compact computational stencil, low CPU cost, and, often, the solution boundness, but have large dissipation and/or dispersion errors. Then such schemes are upgraded to a higher order, e.g., within the framework of 'variable-extrapolation' and 'flux-extrapolation' techniques (e.g., van Leer, 1979; Woodward and Colella, 1984; Drikakis, 2003), by extending the spatial stencil. The upgrade is needed to enhance linear wave propagation properties of the solution away from the flow discontinuities. In the vicinity of high-gradients of the solution, either nonlinear flux limiter (FCT/TVD) functions are used (Boris et al., 1975; Harten et al., 1987; Pietrzak, 1998), or solutionadaptive stencils are used, as in WENO-type schemes (Shu and Osher, 1988). A significant improvement of the linear wave propagation properties can also be obtained by introducing additional degrees of freedom inside each computational cell, in the framework of Discontinuous Galerkin methods (Cockburn and Shu, 2001), which are less sensitive to spatial grid non-uniformity, relative to the high-resolution finite-difference schemes.

Standard second- and third-order non-oscillatory schemes are typically too dissipative for the problems in which the solution-front-sharpening mechanism is either absent or too weak to counterbalance the effect of the numerical dissipation. The higher cost of implementation and the extra CPU cost of a high-order scheme is accepted by the computational community for some situations, when the conventional methods require prohibitively fine grids. One may argue that high-order discretisation in space is the only way to improve the solution, but we take a complimentary view. We argue that in a number of situations it is more efficient to improve the properties of the underlying discretisation by addressing additional properties of the governing (hyperbolic) equations, within the class of second-order schemes. We argue that a hierarchy of high-order schemes is better to be developed from a "low-order" method that is as accurate and efficient as possible. Then the order of the 
method can be systematically increased, consistently in space and time, and the low-dispersive and low-dissipative properties of the original scheme can be improved. Finally, because of their robustness, the second-order methods are still widely used as a "working horse" in many hydrodynamics codes (e.g., for non-uniform grids, treatment of the shocks, ease with boundary conditions, etc.), and their improvement is important.

\section{CABARET method}

\subsection{Linear advection scheme}

To illustrate our ideas on a simple example, a scalar conservation law

$$
\partial_{t} u+\partial_{x} f(u)=0
$$

is considered on a finite-difference grid which is non-uniform in space $x_{i+1}-x_{i}=h_{i+1 / 2}$ and time $t^{n+1}-t^{n}=\tau^{n+1 / 2}$

Fig.1 shows the associated data structure on the non-uniform grid in space and time: the solid circles (labelled with letters) show the location of conserved variable $u$ with fractional $i$-indices and the open circles (labelled with numbers) show the locations of the flux variable $f$ with whole $i$-indices. Our scheme assumes that all variables are known at the $n$th time level, and, then, it takes a half time step using forward-time central approximation,

$$
\frac{u_{C}-u_{E}}{\frac{1}{2} \tau^{n+1 / 2}}+\frac{f_{4}-f_{5}}{h_{i+1 / 2}}=0 .
$$

It is continued with another half time step by using the backward-time central-space approximation,

$$
\frac{u_{A}-u_{C}}{\frac{1}{2} \tau^{n+1 / 2}}+\frac{f_{1}-f_{2}}{h_{i+1 / 2}}=0,
$$


where the fluxes $f_{1}, f_{2}$ are still to be determined. Note, that by adding these two equations, one obtains a conventional flux integral around the cell 1254, with a trapezoidal rule evaluation of the fluxes. By symmetry, the scheme will be second-order accurate for a sufficiently accurate evaluation of $f_{1}$ and $f_{2}$. We choose to evaluate these by a simple upwind extrapolation carried out in an upwind manner. Let's assume for the moment that the sign of the wave speed, $\partial_{u} f(u)$, is positive everywhere. Then, we determine $f_{1}=f\left(u_{1}\right)$ by assuming that

$$
u_{1}=2 u_{C}-u_{5}
$$

With this choice, the entire scheme (2)-(4) is time-reversible. It is also second order accurate, regardless of the gird non-uniformity in space and time, and it is non-dissipative.

The CABARET scheme (2)-(4) is an explicit single-temporal-stage method (note that the upwind extrapolation step for updating the fluxes is applied once per time step). The scheme is conservative

and stable under the Courant (CFL) condition: $0 \leq|c| \tau / h \leq 1$. Due to its compact computational stencil, it remains second order accurate even on non-uniform spatial and temporal grids.

\subsection{Treatment of unresolved short-wave scales in the solution.}

Regardless of how good the numerical scheme is, it is unlikely to correctly resolve all flow scales which can emerge in high-Re flow regimes. A feasible approach is to modify the original nondissipative numerical method so that the small scales are harmlessly removed from the solution, without spurious backscatter from small to large scales (e.g., Pope, 2000; Grinstein, et al., 2007). The discrete grid resolution limit is consistent with the Godunov Theorem (Godunov, 1959) which states that any monotone scheme is first order accurate. This implies for a numerical smoothing procedure to 
be used in the vicinity of unresolved solution gradients. Note that the resolution problem of Eulerian schemes can be directly related to their dispersion errors at high wavenumbers. The better numerical advection scheme is, the closer to the computational grid size is the properly resolved wave, and the better tailored is the use of the numerical smoothing.

For making the CABARET solution non-oscillatory, a simple tunable-parameter-free flux limiter is introduced through a non-linear correction procedure for the flux variables:

$$
\begin{aligned}
& u_{1}=2 u_{C}-u_{5} ; \\
& \text { if }\left(u_{1}>\operatorname{Max}\left(u_{4}, u_{E}, u_{5}\right)\right) u_{1}=\operatorname{Max}\left(u_{4}, u_{E}, u_{5}\right) ; \\
& \text { if }\left(u_{1}<\operatorname{Min}\left(u_{4}, u_{E}, u_{5}\right)\right) u_{1}=\operatorname{Min}\left(u_{4}, u_{E}, u_{5}\right) .
\end{aligned}
$$

The above nonlinear correction procedure is based on the maximum principle (e.g., Boris et al., 1975; Harten et al., 1987) for characteristic wave that arrives at point 1 from the solution domain dependency 4-E-5, and that is approximated using the 3-point stencil $\left(u_{4}, u_{E}, u_{5}\right)$ within one cell in space. In comparison to the standard FCT/TVD schemes, the nonlinear correction algorithm is directly based on enforcing the maximum principle on flux variables, rather than limiting the slopes of conservation variables.

Despite the fact that the correction method (4a) does not explicitly enforce monotonicity on the conservation variables, the nonlinear flux correction allows the CABARET scheme to suppress nonphysical oscillations in the solution. Numerical tests and theoretical investigations (e.g., Ostapenko, 2009) have confirmed that the solution of the CABARET method with flux correction (4a) remains strictly free both from spurious oscillations and "stair-casing" artifacts of the standard TVD schemes (e.g., Shchepetkin and McWilliams, 1998) for all Courant numbers CFL $\leq 0.5$. Fig.2 shows the comparison of the CABARET solution for the linear advection of discontinuous and continuous initial 
data with the second-order van Leer TVD scheme based on MinMod and Superbee limiters (e.g., Hirsch, 2007).

It should be mentioned that there are more sophisticated ways of introducing conservative correction in the CABARET scheme, than the simple limiter (4a). For example, there are algorithms that enforce monotonicity simultaneously to the flux and conservation variables (e.g., Goloviznin et al., 2003). For simple cases, e.g., for linear advection with constant velocity, they produce more accurate results than the simple algorithm (2)-(4a). A particular example is the so-called Digital Transport Algorithm (e.g., Karabasov and Goloviznin, 2004), which leads to the exact solution of 1-D and 2-D advection problems for any piece-wise constant initial data at $0<\mathrm{CFL}<1 \quad(0<\mathrm{CFL}<0.5$ for $2-\mathrm{D}$ problems $)$ on Eulerian grids. Despite our interest in such generalizations of the CABARET method (2)-(4a), they will not be discussed any further in the current publication, since their extension to the ocean modelling is in progress.

\subsection{Comparison with other Leapfrog schemes}

Schemes similar to (2)-(4) exist in the literature; they are the Upwind Leapfrog (UL) schemes first discussed by Iserlis for linear advection (1986), and later developed by Roe (1998), Tran and Scheurer (2002), and Kim (2004) for multidimensional wave propagation. However these schemes were neither conservative, nor based on a compact one-space-cell one-time-step computational stencil. Also, they were not equipped with the limiters enabling them to overcome non-physical oscillations in the solution; hence, they were not robust enough for practical applications. In parallel to these efforts, Goloviznin and Samarskii (1998) proposed an extension of the UL scheme to conservative methods. Later, Goloviznin and co-workers developed CABARET versions with the limiters (Goloviznin and Karabasov, 1998) and extended the method to multidimensional wave dispersion in fracturated porous 
medium (Goloviznin et al. 2007) and gas dynamics (Goloviznin, 2005; Karabasov and Goloviznin, 2007).

To see that the present method is reduced to the UL for the linear advection $(f=c \cdot u, c=c o n s t)$ on a uniform grid in space and time, note that

$$
u_{C}-u_{E}=v\left(u_{5}-u_{4}\right) \text { and } u_{E}-u_{G}=v\left(u_{5}-u_{4}\right)
$$

where $v=c \tau / h$ is the CFL number. Hence,

$$
u_{C}-u_{E}=v\left(u_{5}-u_{4}\right)=\frac{1}{2}\left(u_{1}+u_{5}-u_{4}-u_{8}\right)=\frac{1}{2}\left(\left(u_{1}-u_{4}\right)+\left(u_{5}-u_{8}\right)\right)
$$

which is the three-time-level UL method.

Note, that in comparison to the CABARET stencil (1-A-2-C-4-E-5) the UL method (1-4-5-8) uses a double-interval computational stencil in time, whereas the conventional Central Leapfrog (CL) method

$$
v\left(u_{6}-u_{4}\right)=\left(u_{2}-u_{8}\right)
$$

uses computational stencil which is twice as wide as that of the UL scheme.

In addition to removing the defects of the UL, the CABARET scheme retains all of the UL good properties, such as ease of implementation near boundaries, zero numerical dissipation and very small dispersion error.

An important distinction between the implementations of the CABARET and UL schemes is that the UL scheme is three-time-level and needs to be properly initialized, otherwise a spurious mode can be excited. CABARET provides unambiguous prediction at level $(n+1)$ from data at level (n). However, the same spurious mode can be excited if at the initial time step the flux data are not consistent with the conservation variables. 
An important feature of the CABARET/UL scheme is that it approximates the entire material derivative on the Eulerian grid:

$$
L=\left\langle\partial_{t}+\partial_{u} f(u) \partial_{x}\right\rangle
$$

rather than splits it into independent, space and time components

$$
L_{x}=\left\langle\partial_{u} f(u) \cdot \partial_{x}\right\rangle, L=\left\langle\partial_{t}\right\rangle+L_{x}
$$

Note that separate space and time treatment (9) is used in many high-resolution schemes based on highorder spatial-flux reconstruction techniques, combined with time-integration methods based on lowdissipative multi-step Runge-Kutta techniques (e.g., Bogey and Bailly, 2004) or on single-step predictor-corrector methods of Adams-type (e.g., Shchepetkin and McWilliams, 1998). In particular, we argue that in comparison to the methods based on the Adams-type schemes, the CABARET scheme (2)-(4) is non-dissipative, which makes it more suitable for turbulent flow regimes. A comparison of the dispersion properties of the CABARET/ UL scheme with those of the central finite differences, including optimised high-order schemes typically used with low-dissipative Runge-Kutta timeintegration schemes, is in the next section.

\subsection{Results of linear dispersion analysis}

The non-staggered form of finite-difference schemes (without nonlinear flux correction) is well suited for analysis of the linear-wave dissipation and dispersion properties. In this analysis a travelling wave solution, $\varphi_{m}^{n} \square \exp [i \omega \cdot \tau \cdot n-i k \cdot h \cdot m]$, is substituted into the scheme. The resulting algebraic equation is solved for the frequency, $\omega=\omega(k \cdot h)$, or $\frac{\omega \cdot \tau}{c \cdot k \cdot h}=f(k \cdot h, C F L)$, where $f$ is a function of the dimensionless wavenumber, $k \cdot h$, and of the Courant number, $C F L=|c| \tau / h$. The outcome is used 


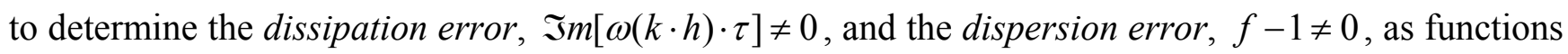
of the wavenumber and the Courant number. In Fig.3 we compare the dispersion error of the CABARET scheme at different Courant numbers against the errors of semi-discrete finite differences including two state-of-the art high-order low-dispersion schemes used in turbulence modeling (e.g., Lele, 1992). Note, that the dispersion properties of semi-discrete schemes correspond to exact integration in time, that neglects possible increase of the dispersion error due to inaccuracies in time marching, and that is equivalent to using a multi-stage Runge-Kutta method with a very small step for time integration. We find that for most Courant numbers and for a wide range of grid resolution (7-20 points per wavelength) the dispersion error of the CABARET scheme remains below that of the conventional and optimised fourth-order central finite differences and close to that of the six-order central schemes. Away from the optimal Courant number range $(\mathrm{CFL}=0.1)$, the $\mathrm{CABARET}$ dispersion error is similar to that of the fourth-order scheme. Within this range of grid resolution the error decay rate (error slope) of the CABARET solution is in between the forth- and the second-order schemes.

Another important property pertaining to the numerical dispersion error is the numerical group speed (normalized by the advection speed), $c_{g}=\frac{\tau}{c} \frac{\partial(\omega(k \cdot h))}{\partial(k \cdot h)}$. Any deviation of $c_{g}$ from 1 corresponds to an error in physical wave propagation. In particular, negative values of $c_{g}$ lead to spurious wave reflection. In this case, all waves starting from a certain frequency are "grid locked" and their energy is back-scattered (Colonius and Lele, 2004). Fig.4 shows the numerical group speeds of the same finite-difference schemes as in Fig.3. It can be seen that all central schemes, including the high-order compact Pade-type scheme, have $c_{g}<0$ for short enough wavelengths. In contrast, the CABARET group speed remains always positive, and the waves propagate at all frequencies. The 
magnitude of the CABARET $c_{g}$ error is small: even for CFL $=0.1$, it is similar to that of the fourthorder central differences in a wide range of wavenumbers $(0.2<k \cdot h / \pi<0.8)$.

\subsection{CABARET extension for linear advection in multiple dimensions}

Let us consider a linear advection equation in multiple dimensions:

$$
\frac{\partial}{\partial t} u+\sum_{k} \frac{\partial}{\partial x_{k}} f_{k}=0, f_{k}=c_{k} u, c_{k}=\text { const }>0 .
$$

For solving this equation with the CABARET scheme on a quadrilateral/hexagonal, possibly multiple-block, Cartesian grid, first, we introduce the conservation variables, $u$, referred to the cell centres, and the flux variables, $f_{k}$, referred to the cell face centres in each coordinate direction, $1 \leq k \leq d$, where $d=\overline{1 . .3}$ is the dimension of the problem.

Fig.5 shows the assumed data structure for case $d=2$, within one cell in space. Solid circles labelled with letters show locations of the conserved variable, and open circles labelled with numbers show locations of the flux variable, same as for the 1-D CABARET scheme shown in Fig.1. Then, the CABARET algorithm becomes:

Predictor step,

$$
\frac{u_{C}-u_{E}}{\frac{1}{2} \tau^{n+1 / 2}}+\sum_{k} \frac{\partial}{\partial x_{k}} f_{k}^{n}=0
$$

Extrapolation step,

$$
u_{1, k}=2 u_{C}-u_{5, k}, \quad 1 \leq \mathrm{k} \leq d
$$

Corrector step, 


$$
\frac{u_{A}-u_{C}}{\frac{1}{2} \tau^{n+1 / 2}}+\sum_{k} \frac{\partial}{\partial x_{k}} f_{k}^{n+1}=0
$$

Here, the divergence terms are approximated using the flux CABARET variables defined within the same cell, with the second-order accuracy in space. The multidimensional CABARET scheme (11)(13) is second order accurate in time and space, similar to the 1-D CABARET scheme (7)-(9). The multidimensional CABARET scheme is stable for $0<\mathrm{CFL}<1 / \mathrm{d}$. For large-gradient flows, the nonlinear flux correction is applied at the extrapolation step (compare with (15)):

$$
\begin{aligned}
& u_{1, k}=2 u_{C}-u_{5, k} ; \\
& M=\max \left(u_{4, k}, u_{E}, u_{5, k}\right)+\int_{t=t^{n}}^{t=t^{n+1}} Q(t) d t ; \quad m=\min \left(u_{4, k}, u_{E}, u_{5, k}\right)+\int_{t=t^{n}}^{t=t^{n+1}} Q(t) d t ; \\
& Q(t)=-\sum_{i \neq k} \frac{\partial}{\partial x_{i}} f_{i} ; \\
& \text { If } u_{1, k}>M, \quad u_{1, k}=M ; \\
& \text { If } u_{1, k}<m, \quad u_{1, k}=m .
\end{aligned}
$$

Thus, the multidimensional CABARET scheme updates each flux variable by performing 1D extrapolation separately for each coordinate direction, but uses the same conservation variable for all directions. Thus, it combines the simplicity of the dimension-by-dimension splitting approach with genuinely multidimensional advection treatment.

For demonstration, the classical solid body rotation test (Crowley, 1968) is considered, in which the initial cone distribution is specified on a uniform Cartesian grid in the centre of $1 / 4$ of square computational domain. The advection velocity field corresponds to a steady rotation around the computational domain centre. The vertex of the cone is a singularity, and preserving it numerically is rather challenging. Fig. 6 shows that after one revolution on a coarse grid (20 points per the cone diameter), the cone symmetry is still well preserved. This solution corresponds to the relative cone 
vertex clipping of $16 \%$ which is twice smaller than $34 \%$ vertex reduction reported by Hundsdorfer et al. (1995) for the third-order MUSCL TVD scheme.

\subsection{Example of CABARET scheme for the system of hyperbolic equations}

The CABARET method has been extended to the compressible Euler equations with the use of characteristic decomposition. Standard characteristic Eulerian methods are based on flow field decomposition into simple characteristic waves of constant amplitudes obtained from the solution of 1D Riemann problem at each cell face (e.g., LeVeque, 2002; Hirsh, 2007). In contrast to those, the CABARET extension to the equations in primitive variables is based on the extrapolation of groups of characteristic variables, i.e., local Riemann invariants. In particular, the solution algorithm for the 1-D gas dynamics is outlined below.

The components of the conservative variables are updated at the predictor and corrector step (Fig.1):

$$
\begin{aligned}
& \frac{\mathbf{U}_{C}-\mathbf{U}_{E}}{\frac{1}{2} \tau^{n+1 / 2}}+\frac{\mathrm{F}\left(\mathbf{U}_{4}\right)-\mathrm{F}\left(\mathbf{U}_{5}\right)}{h_{i+1 / 2}}=0 ; \\
& \frac{\mathbf{U}_{A}-\mathbf{U}_{C}}{\frac{1}{2} \tau^{n+1 / 2}}+\frac{\mathrm{F}\left(\mathbf{U}_{1}\right)-\mathrm{F}\left(\mathbf{U}_{2}\right)}{h_{i+1 / 2}}=0 ; \\
& \mathbf{U}=(\rho, \rho u, E)^{T}, \mathbf{F}=\left(\rho u, \rho u^{2}+p, u(E+p)\right)^{T},
\end{aligned}
$$

where $\rho, \rho u, E$ are density, momentum and energy, respectively.

At the extrapolation step the flux variables are computed at cell faces. In order to update the flux variable on the new time level at cell face $(i)$, we use local Riemann invariants that are referred to each cell centre, left, $(i-1 / 2)$, and right, $(i+1 / 2)$, from the computational node $(i)$. For example, for ideal gas flows the following form of the local Riemann invariants referred to the space-time cell $(i+1 / 2$, $\mathrm{n}+1 / 2$ ) can be used:

$$
\mathbf{R}\left(\rho, u, p \mid S_{C}\right)=\left(u+A p^{\mu}, u-A p^{\mu}, \ln \left(p \cdot \rho^{-\gamma}\right)\right)^{\mathrm{T}}, A=\frac{2 \sqrt{\gamma}}{\gamma-1}\left(S_{C}\right)^{\frac{1}{2 \gamma}}, \mu=\frac{\gamma-1}{2 \gamma},
$$

where $S_{C}=p_{C} \cdot\left(\rho_{C}\right)^{-\gamma}$ is the cell-based entropy variable. The three components of the characteristic vector correspond to eigenvalues $\left\{\lambda_{q}\right\}_{C}=\{u+a, u-a, u\}_{C}, q=\overline{1 . .3}$, where $a$ is sound speed and $\gamma$ is 
specific heat ratio. For locally isentropic flows, this choice of characteristic invariants allows increasing the solution accuracy during the extrapolation stage. For each computational cell face $(i)$, we compute its neighbouring local Riemann invariants (16) defined in cells $(i-1 / 2)$ and $(i+1 / 2)$, according to the CABARET data structure shown in Fig.1. For example, for point 2 this involves computation of the local Riemann invariants in points 4,5,6 and C,D,E,F. The extrapolation of local Riemann invariants from cells (1-2-4-5) and (2-3-5-6) to point 2 results in two sets of local Riemann invariants. Once the local Riemann invariants are obtained, their values are subject to the conservative flux correction (4a). From the two vectors of characteristic variables a single conservative flux vector is reconstructed by using only those components of each vector that correspond to incoming characteristics to the cell face. Note that the use of a double set of local Riemann invariants is analogous to that based on using a double characteristic Jacobian matrix that is known to increase the robustness of characteristic decomposition techniques (Donat and Marquina, 1996).

As a demonstration of the CABARET approach capabilities, we consider the so-called ShuOsher problem (Shu and Osher, 1988), in which a shock wave of Mach 3 interacts with a stationary entropy wave:

$$
\begin{aligned}
& (\rho, u, p)=\left\{\begin{array}{c}
(3.857143,2.629369,10.333333) \text { for } \mathrm{x}<2 ; \\
(1+0.2 \sin (5 x), 0,1) \text { for } \mathrm{x} \geq 2 ;
\end{array}\right. \\
& x \in[0,10] .
\end{aligned}
$$

The test is challenging for it requires from the numerical method not only to be shock-capturing but also to preserve the rich solution structure behind the shock front. Table 1 shows the comparison of the CABARET solution with other high-resolution methods considered by Woodward and Colella (1984). Fig.7 shows the instantaneous density field for the CABARET solution at a longer time $t=1.872$ that corresponds to the grid resolution of 400 cells.

The numerical errors and total number of time iterations are summarized in Table 1. The total number of iterations corresponds to the number of time steps $(N T)$ required to reach the control time moment, $\mathrm{t}=1.8$. The numerical error is defined as a relative error based on the following norms: 


$$
\varepsilon_{1}=\frac{1}{N+1} \sum \frac{\left|\rho-\rho_{\text {exact }}\right|}{\left|\rho_{\text {exact }}\right|}, \quad \varepsilon_{c}=\operatorname{Max}\left(\frac{\left|\rho-\rho_{\text {exact }}\right|}{\left|\rho_{\text {exact }}\right|}\right) .
$$

Table 1. Errors for the shock/density wave interaction problem

\begin{tabular}{|l|l|l|l|l|l|l|l|l|r|}
\hline & \multicolumn{3}{|c|}{ PLIMDE } & \multicolumn{3}{c|}{ WENO } & \multicolumn{3}{c|}{ CABARET } \\
\hline $\mathrm{N}$ & $\mathrm{NT}$ & $\varepsilon_{1}$ & $\varepsilon_{\mathrm{c}}$ & $\mathrm{NT}$ & $\varepsilon_{1}$ & $\varepsilon_{\mathrm{c}}$ & NT & $\varepsilon_{1}$ & $\varepsilon_{\mathrm{c}}$ \\
\hline 200 & 250 & 0.0185 & 0.429 & 334 & 0.0214 & 0.902 & $\mathbf{2 2 9}$ & $\mathbf{0 . 0 2 1 9}$ & $\mathbf{0 . 1 2 1 1}$ \\
\hline 400 & 468 & 0.0183 & 0.188 & 634 & 0.0195 & 0.667 & $\mathbf{4 6 2}$ & $\mathbf{0 . 0 1 0 0}$ & $\mathbf{0 . 1 4 6 7}$ \\
\hline 800 & 903 & 0.0166 & 0.290 & 1235 & 0.0162 & 0.369 & $\mathbf{9 2 7}$ & $\mathbf{0 . 0 0 5 8}$ & $\mathbf{0 . 2 4 0 0}$ \\
\hline
\end{tabular}

WENO is the $3^{\text {rd }}$ order Weighted Essentially Non Oscillatory Scheme and PLIMDE is

the second-order Parabolic Interpolation Method.

As follows from the table, the maximum error in the $\varepsilon_{c}-$ norm remains smallest for the CABARET scheme, among all three schemes. In contrast to the other schemes, in the $\varepsilon_{1}-$ norm, the CABARET solution error leads to the convergence rate which is higher than 1 for all grids, including the coarsest one.

\section{Application to the double-gyre circulation}

In this section we apply the CABARET approach to a classical ocean circulation problem.

\subsection{Ocean model}

We keep in mind ultimately applying the CABARET approach to a fully comprehensive, primitive-equation, eddy-resolving general circulation model of the ocean, but, for the purpose of this paper, we focus on the more simple QG ocean dynamics. The QG model of the wind-driven double- 
gyre circulation is considered in a midlatitude closed basin, which is in the shape of a square with north-south and east-west rigid walls $([L \times L]=3840 \times 3840 \mathrm{~km})$. This model simulates not only the subpolar and subtropical ocean gyres but also the nonlinear western boundary currents, such as the Gulfstream or Kurosio, and their eastward jet extensions (e.g., Holland, 1978). The model stratification is represented by 3 stacked isopycnal layers that are dynamically coupled through pressure fluctuations. The governing equations constitute the system of material conservation laws for potential vorticity (PV) anomaly, $\zeta_{q}, q=\overline{1 . .3}$, with a source term due to the meridional gradient of the Coriolis parameter and with the additional source terms due to the lateral viscosity, bottom friction, and the wind forcing. The system of the quasilinear hyperbolic-type (e.g., Rozhdestvensky and Yanenko, 1978) equations for PV anomaly and the associated elliptic equations for velocity streamfunctions are:

$$
\begin{aligned}
& \frac{\partial}{\partial t} \zeta_{q}+\frac{\partial}{\partial x}\left(u_{q} \zeta_{q}\right)+\frac{\partial}{\partial y}\left(v_{q} \zeta_{q}\right)=F_{q}, \quad q=\overline{1 . .3}, \\
& F_{q}=\delta_{1 q} \cdot f_{\text {wind }}-\beta \cdot v_{q}+\delta_{3 q} \cdot \mu_{b o t} \nabla^{2} \psi_{q}+\mu_{e d d y} \nabla^{2} D_{q}, \\
& u_{q}=\frac{\partial \psi_{q}}{\partial y}, \quad v_{q}=-\frac{\partial \psi_{q}}{\partial x}, \\
& \zeta_{1}=\nabla^{2} \psi_{1}-s_{1}\left(\psi_{1}-\psi_{2}\right), D_{1}=\zeta_{1}+s_{1}\left(\psi_{1}-\psi_{2}\right), \\
& \zeta_{2}=\nabla^{2} \psi_{2}-s_{21}\left(\psi_{2}-\psi_{1}\right)-s_{22}\left(\psi_{2}-\psi_{3}\right), D_{2}=\zeta_{2}+s_{21}\left(\psi_{2}-\psi_{1}\right)+s_{22}\left(\psi_{2}-\psi_{3}\right), \\
& \zeta_{3}=\nabla^{2} \psi_{3}-s_{3}\left(\psi_{3}-\psi_{2}\right), D_{3}=\zeta_{3}+s_{3}\left(\psi_{3}-\psi_{2}\right), \\
& \beta, \mu_{b o t}, \mu_{e d d y}, s_{1}, s_{21}, s_{22}, s_{3}=\text { const }>0,
\end{aligned}
$$

where $q=1,2$, and 3 denote the top, intermediate, and the bottom isopycnal layers, respectively; $\delta_{k q}$ is the Kronecker symbol; $\beta$ is the meridional gradient of the Coriolis parameter; $f_{\text {wind }}=f_{\text {wind }}(x, y)$ is the idealised steady forcing proportional to the wind stress curl: 


$$
\begin{aligned}
& f_{\text {wind }}(x, y)=\left\{\begin{array}{l}
A \sin \left(\frac{\pi \cdot y / L}{y_{0} / L}\right) \quad \text { for } 0 \leq y<y_{0}, \\
-A \sin \left(\frac{\pi \cdot\left(y-y_{0}\right) / L}{1-y_{0} / L}\right) \text { for } y_{0} \leq y \leq L,
\end{array}\right. \\
& y_{0} / L=0.5+0.2 \cdot(x / L-0.5), \quad A=-2 \pi \tau_{0} /\left(0.9 \cdot L \cdot \rho_{1}\right) ;
\end{aligned}
$$

where the wind stress amplitude is $\tau_{0}=0.8 \mathrm{~N} \cdot \mathrm{m}^{-2}$, and the upper-ocean density is $\rho_{1}=10^{3} \mathrm{~kg} / \mathrm{m}^{3}$ (e.g., Berloff et al., 2007). The wind forcing is chosen to be asymmetric and tilted with respect to the middle latitude of the basin, in order to avoid the artificial symmetrisation of the gyres. The layer depths, from top to bottom, are $H_{1}=0.25, H_{2}=0.75$, and $H_{3}=3 \mathrm{~km}$, respectively. The stratification parameters, $s_{1}, s_{21}, s_{22}, s_{3}$, are chosen so that the first and the second internal deformation radii are 32.2 and $18.9 \mathrm{~km}$, respectively. Here, $\mu_{\text {bot }}$ and $\mu_{\text {eddy }}$ are coefficients of the bottom friction and the lateral eddy viscosity, respectively. For the benchmark solutions we use $\mu_{\text {eddy }}=12$ and $50 \mathrm{~m}^{2} \mathrm{~s}^{-1}$, and $\mu_{b o t}=4 \cdot 10^{-8} s^{-1}$.

The larger value of $\mu_{\text {eddy }}$ corresponds to a Reynolds number, $\operatorname{Re}=U \cdot L \cdot \mu_{\text {eddy }}{ }^{-1} \approx 3200$, where $U=\tau_{0}\left(\rho_{1} \cdot H_{1} \cdot L \cdot \beta\right)^{-1}=0.0417 \mathrm{~m} / \mathrm{s}$ is the linear-dynamics velocity scale given by the balance between the $\beta$-term and the wind forcing.

It is commonly assumed that the eddy viscosity (or eddy diffusion) crudely parameterises unresolved effects of the nonlinear eddies. However, due to the "negative viscosity" phenomena (Starr, 1965), the effects of the mesoscale eddies are not always described by the diffusion, therefore, it is highly desirable to run ocean models in eddy-resolving regimes. Such regimes can be achieved by a systematic reduction of the eddy viscosity, hence, by a systematic increase of Re, until the nonlinear eddies are dynamically permitted. Solving for high-Re regimes needs to be backed up by using finer grid resolution and more accurate numerical algorithms. 
For the flow regions where the flow characteristics do not intersect (e.g., away from the shocks), the conservative form of the governing equations can be rewritten in the following $1 \mathrm{D}$ characteristic-wave form:

$$
\begin{array}{ll}
\left(\frac{\partial}{\partial t}+u_{q} \frac{\partial}{\partial x}\right) \zeta_{q}=Q_{q}^{(u)}, & \left(\frac{\partial}{\partial t}+v_{q} \frac{\partial}{\partial y}\right) \zeta_{q}=Q_{q}^{(v)}, \quad q=\overline{1 . .3}, \\
Q_{q}^{(u)}=F_{q}-v_{q} \frac{\partial}{\partial y} \zeta_{q}, & Q_{q}^{(v)}=F_{q}-u_{q} \frac{\partial}{\partial x} \zeta_{q},
\end{array}
$$

where the notations are the same as in (19). This form emphasises the characteristic behaviour of the PV anomaly, as if it is a scalar-transported tracer in a non-uniform divergence-free velocity field. At the closed-basin boundary, $\Gamma$, the partial-slip condition is imposed:

$$
\left.\left(u_{q} n_{x}+v_{q} n_{y}\right)\right|_{\Gamma}=0,\left.\left(\frac{\partial}{\partial n}\left(u_{q} n_{x}+v_{q} n_{y}\right)-\alpha^{-1} \cdot\left(u_{q} n_{x}+v_{q} n_{y}\right)\right)\right|_{\Gamma}=0, \quad \alpha=\text { const }>0, \quad q=\overline{1 . .3},(21)
$$

where $\left(n_{x}, n_{y}\right)$ are the Cartesian components of the normal unit vector. This boundary condition implies that the tangential velocity component at the wall corresponds to a prescribed exponentialdecay (i.e., 'wall-function') law based on the characteristic boundary layer thickness, $\alpha$. This condition corresponds to the mixed Dirichlet-Neumann boundary condition (e.g., Hirsch, 2007). In the ocean modelling practice, it is a conventional, though not fully justified, parameterisation for dynamically unresolved processes near the ocean coasts. In our numerical algorithm, we apply (21) in the velocity-streamfunction form (normal-to-wall derivatives are taken):

$$
\left(\left(\psi_{q}\right)^{\prime \prime}-\frac{\left(\psi_{q}\right)^{\prime}}{\alpha}\right)_{\Gamma}=0, q=\overline{1 . .3}
$$

and we use $\alpha=120 \mathrm{~km}$.

Together with the integral mass conservation constraints (McWilliams, 1977), 


$$
\frac{\partial}{\partial t} \iint\left(\psi_{1}-\psi_{2}\right) d x d y=0 \text { and } \frac{\partial}{\partial t} \iint\left(\psi_{2}-\psi_{3}\right) d x d y=0
$$

the boundary condition (22) is a part of the elliptic-problem inversion of PV anomaly, $\zeta_{q}$, into the velocity streamfunctions.

\subsection{Numerical method based on the CABARET scheme}

In this section we introduce CABARET method to the ocean model described in Section 3.1.

Let us consider a uniform Cartesian grid, $x_{i+1, j}-x_{i, j}=h, y_{i, j+1}-y_{i, j}=h, 1 \leq i, j \leq N$, which covers the entire computational domain. In addition to this grid, we consider the nodes staggered from it by $h / 2$ :

$$
\begin{array}{cc}
x_{i+1 / 2, j+1 / 2}=x_{i, j}+h / 2, & y_{i+1 / 2, j+1 / 2}=y_{i, j}+h / 2 ; \\
x_{i, j+1 / 2}=x_{i, j}, & y_{i, j+1 / 2}=y_{i, j}+h / 2 ; \\
x_{i+1 / 2, j}=x_{i, j}+h / 2, & y_{i+1 / 2, j}=y_{i, j} .
\end{array}
$$

The conservation variables of the CABARET scheme are referred to the cell centre points (fractional $i$ and $j$ indices); the flux variables in the x-direction are referred to the cell face centres (whole $i$-indices); and the flux variables in the y-direction are referred to the cell face centres (whole $j$-indices).

The extension of the 1D CABARET algorithm (7)-(9) for the conservation law (19) is the following. At the predictor step, the PV anomaly value is computed at the mid-time level:

$$
\begin{aligned}
& \frac{\left(\zeta_{q}\right)_{i+1 / 2, j+1 / 2}^{n+1 / 2}-\left(\zeta_{q}\right)_{i+1 / 2, j+1 / 2}^{n}}{\tau^{n+1 / 2} / 2}+\frac{\left(u_{q}\right)_{i+1, j+1 / 2}^{n}\left(\zeta_{q}\right)_{i+1, j+1 / 2}^{n}-\left(u_{q}\right)_{i, j+1 / 2}^{n}\left(\zeta_{q}\right)_{i, j+1 / 2}^{n}}{h}+ \\
& \quad+\frac{\left(v_{q}\right)_{i+1 / 2, j+1}^{n}\left(\zeta_{q}\right)_{i+1 / 2, j+1}^{n}-\left(v_{q}\right)_{i+1 / 2, j}^{n}\left(\zeta_{q}\right)_{i+1 / 2, j}^{n}}{h}=\left(F_{q}\right)_{i+1 / 2, j+1 / 2}^{n+1 / 2}, \quad q=\overline{1 . .3}
\end{aligned}
$$


where $\left(F_{q}\right)_{i+1 / 2, j+1 / 2}^{n+1 / 2}$ denotes the second-order accurate approximation (discussed later in this section) of the source function at the mid-time level; and the minimal local step, according to the stability constraint, is

$$
\tau^{n+1 / 2}=\operatorname{Min}_{1 \leq i, j \leq N}\left[C F L \cdot h / \operatorname{Max}\left(\left|\left(u_{q}\right)_{i, j+1 / 2}^{n}\right|,\left|\left(v_{q}\right)_{i+1 / 2, j}^{n}\right|\right)\right],
$$

where $0<C F L<0.5$. The updated values of PV anomaly are used to compute the mid-time-level velocity streamfunction and its Laplacian, by solving the system of the elliptic equations,

$$
\begin{aligned}
& \left(\zeta_{1}\right)_{i+1 / 2, j+1 / 2}^{n+1 / 2}=\left(\nabla^{2} \psi_{1}\right)_{i+1 / 2, j+1 / 2}^{n+1 / 2}-S_{1}\left(\left(\psi_{1}\right)_{i+1 / 2, j+1 / 2}^{n+1 / 2}-\left(\psi_{2}\right)_{i+1 / 2, j+1 / 2}^{n+1 / 2}\right), \\
& \left(\zeta_{2}\right)_{i+1 / 2, j+j+1 / 2}^{n+1 / 2}=\left(\nabla^{2} \psi_{2}\right)_{i+1 / 2, j+j+1 / 2}^{n+1 / 2}-S_{21}\left(\left(\psi_{2}\right)_{i+1 / 2, j+1 / 2}^{n+1 / 2}-\left(\psi_{1}\right)_{i+1 / 2, j+1 / 2}^{n+1 / 2}\right)-S_{22}\left(\left(\psi_{2}\right)_{i+1 / 2, j+1 / 2}^{n+1 / 2}-\left(\psi_{3}\right)_{i+1 / 2, j+1 / 2}^{n+1 / 2}\right), \\
& \left(\zeta_{3}\right)_{i+1 / 2, j+1 / 2}^{n+1 / 2}=\left(\nabla^{2} \psi_{3}\right)_{i+1 / 2, j+1 / 2}^{n+1 / 2}-S_{3}\left(\left(\psi_{3}\right)_{i+1 / 2, j+1 / 1 / 2}^{n+1 / 2}-\left(\psi_{2}\right)_{i+1 / 2, j+1 / 2}^{n+1 / 2}\right),
\end{aligned}
$$

complemented by the partial-slip boundary condition (22) approximated with the second-order onesided finite-differences and by the imposed mass conservation constraint (23).

Once $\left(\psi_{q}\right)_{i+1 / 2, j+1 / 2}^{n+1 / 2}$ is calculated, it is used for updating the velocity components at the cell faces:

$$
\begin{aligned}
& \left(\psi_{q}\right)_{i, j}^{n+1 / 2}=\frac{1}{4}\left(\left(\psi_{q}\right)_{i+1 / 2, j+1 / 2}^{n+1 / 2}+\left(\psi_{q}\right)_{i+1 / 2, j-1 / 2}^{n+1 / 2}+\left(\psi_{q}\right)_{i-1 / 2, j+1 / 2}^{n+1 / 2}+\left(\psi_{q}\right)_{i-1 / 2, j-1 / 2}^{n+1 / 2}\right), \\
& \left(u_{q}\right)_{i, j+1 / 2}^{n+1 / 2}=\frac{\left(\psi_{q}\right)_{i, j+1}^{n+1 / 2}-\left(\psi_{q}\right)_{i, j}^{n+1 / 2}}{h},\left(v_{q}\right)_{i+1 / 2, j}^{n+1 / 2}=-\frac{\left(\psi_{q}\right)_{i+1, j}^{n+1 / 2}-\left(\psi_{q}\right)_{i, j}^{n+1 / 2}}{h} .
\end{aligned}
$$

Then, each velocity component is extrapolated to the new time level, $n+1$, with the second-order accuracy: 


$$
\begin{aligned}
& \left(u_{q}\right)_{i, j+1 / 2}^{n+1}=(1+\delta)\left(u_{q}\right)_{i, j+1 / 2}^{n+1 / 2}-\delta\left(u_{q}\right)_{i, j+1 / 2}^{n-1 / 2}, \\
& \left(v_{q}\right)_{i, j+1 / 2}^{n+1}=(1+\delta)\left(v_{q}\right)_{i, j+1 / 2}^{n+1 / 2}-\delta\left(v_{q}\right)_{i, j+1 / 2}^{n-1 / 2}, \\
& \delta=\frac{\tau^{n+1 / 2}}{\tau^{n+1 / 2}+\tau^{n-1 / 2}} .
\end{aligned}
$$

Once the velocity field is updated, the extrapolation step yields the new values of the flux variables:

$$
\begin{aligned}
& \left(\zeta_{q}\right)_{i+1, j+1 / 2}^{n+1}=2\left(\zeta_{q}\right)_{i+1 / 2, j+1 / 2}^{n+1 / 2}-\left(\zeta_{q}\right)_{i, j+1 / 2}^{n} \text { if }\left(u_{q}\right)_{i+1, j+1 / 2}^{n+1} \geq 0, \\
& \left(\zeta_{q}\right)_{i, j+1 / 2}^{n+1}=2\left(\zeta_{q}\right)_{i+1 / 2, j+1 / 2}^{n+1 / 2}-\left(\zeta_{q}\right)_{i+1, j+1 / 2}^{n} \text { if }\left(u_{q}\right)_{i, j+1 / 2}^{n+1}<0,
\end{aligned}
$$

and

$$
\begin{aligned}
& \left(\zeta_{q}\right)_{i+1 / 2, j+1}^{n+1}=2\left(\zeta_{q}\right)_{i+1 / 2, j+1 / 2}^{n+1 / 2}-\left(\zeta_{q}\right)_{i+1 / 2, j}^{n} \text { if }\left(v_{q}\right)_{i+1 / 2, j+1}^{n+1} \geq 0, \\
& \left(\zeta_{q}\right)_{i+1 / 2, j}^{n+1}=2\left(\zeta_{q}\right)_{i+1 / 2, j+1 / 2}^{n+1 / 2}-\left(\zeta_{q}\right)_{i+1 / 2, j+1}^{n} \text { if }\left(v_{q}\right)_{i+1 / 2, j}^{n+1}<0 .
\end{aligned}
$$

At the lateral boundary cells, the upstream cell-face values are not available, because the partial-slip boundary condition is imposed on the corresponding centre-cell variables. Therefore, we use the firstorder upwind approximation, e.g.:

$$
\left(\zeta_{q}\right)_{1, j+1 / 2}^{n+1}=\left(\zeta_{q}\right)_{1 / 2, j+1 / 2}^{n+1 / 2} \text { if }\left(u_{q}\right)_{1, j+1 / 2}^{n+1} \geq 0
$$

The computed cell-face values of the PV anomaly are corrected ${ }^{1}$, if they are outside the limits given by the maximum principle:

\footnotetext{
${ }^{1}$ Note that without the flux correction (35),(36) the CABARET solution of the double-gyre problem diverges, same as the Arakawa method without the Robert-Asselin filtering in time.
} 


$$
\begin{aligned}
& \text { If }\left(\zeta_{q}\right)_{i, j+1 / 2}^{n+1}>\left(M_{q}\right)_{i, j+1 / 2}^{n+1}, \quad\left(\zeta_{q}\right)_{i, j+1 / 2}^{n+1}=\left(M_{q}\right)_{i, j+1 / 2}^{n+1}, \\
& \text { If }\left(\zeta_{q}\right)_{i, j+1 / 2}^{n+1}<\left(m_{q}\right)_{i, j+1 / 2}^{n+1}, \quad\left(\zeta_{q}\right)_{i, j+1 / 2}^{n+1}=\left(m_{q}\right)_{i, j+1 / 2}^{n+1},
\end{aligned}
$$

and

$$
\begin{aligned}
& \text { If }\left(\zeta_{q}\right)_{i+1 / 2, j}^{n+1}>\left(M_{q}\right)_{i+1 / 2, j}^{n+1}, \quad\left(\zeta_{q}\right)_{i+1 / 2, j}^{n+1}=\left(M_{q}\right)_{i+1 / 2, j}^{n+1}, \\
& \text { If }\left(\zeta_{q}\right)_{i+1 / 2, j}^{n+1}<\left(m_{q}\right)_{i+1 / 2, j}^{n+1}, \quad\left(\zeta_{q}\right)_{i+1 / 2, j}^{n+1}=\left(m_{q}\right)_{i+1 / 2, j}^{n+1} .
\end{aligned}
$$

Here, the minimum and maximum limit values account not only for the solution at the previous time step, as in (15), but also for the non-zero right-hand side in (19a). Thus, for a cell facing in the $x$ direction, we have:

$$
\operatorname{if}\left(u_{q}\right)_{i+1, j+1 / 2}^{n+1} \geq 0\left\{\begin{array}{l}
\left(M_{q}\right)_{i+1, j+1 / 2}^{n+1}=\operatorname{Max}\left(\left(\zeta_{q}\right)_{i, j+1 / 2}^{n},\left(\zeta_{q}\right)_{i+1 / 2, j+1 / 2}^{n},\left(\zeta_{q}\right)_{i+1, j+1 / 2}^{n}\right)+\left(Q_{q}^{(u)}\right)_{i+1 / 2, j+1 / 2}^{n} \cdot \tau^{n+1 / 2} \\
\left(m_{q}\right)_{i+1, j+1 / 2}^{n+1}=\operatorname{Min}\left(\left(\zeta_{q}\right)_{i, j+1 / 2}^{n},\left(\zeta_{q}\right)_{i+1 / 2, j+1 / 2}^{n},\left(\zeta_{q}\right)_{i+1, j+1 / 2}^{n}\right)+\left(Q_{q}^{(u)}\right)_{i+1 / 2, j+1 / 2}^{n} \cdot \tau^{n+1 / 2}
\end{array}\right.
$$

and

$$
\operatorname{if}\left(u_{q}\right)_{i, j+1 / 2}^{n+1}<0\left\{\begin{array}{l}
\left(M_{q}\right)_{i, j+1 / 2}^{n+1}=\operatorname{Max}\left(\left(\zeta_{q}\right)_{i, j+1 / 2}^{n},\left(\zeta_{q}\right)_{i+1 / 2, j+1 / 2}^{n},\left(\zeta_{q}\right)_{i+1, j+1 / 2}^{n}\right)+\left(Q_{q}^{(u)}\right)_{i+1 / 2, j+1 / 2}^{n} \cdot \tau^{n+1 / 2} \\
\left(m_{q}\right)_{i, j+1 / 2}^{n+1}=\operatorname{Min}\left(\left(\zeta_{q}\right)_{i, j+1 / 2}^{n},\left(\zeta_{q}\right)_{i+1 / 2, j+1 / 2}^{n},\left(\zeta_{q}\right)_{i+1, j+1 / 2}^{n}\right)+\left(Q_{q}^{(u)}\right)_{i+1 / 2, j+1 / 2}^{n} \cdot \tau^{n+1 / 2}
\end{array}\right.
$$

where the source term is evaluated by approximating (19a) with the first-order accuracy in time and with the second-order accuracy in space:

$$
\left(Q_{q}\right)_{i+1 / 2, j+1 / 2}^{n+1 / 2}=\frac{\left(\zeta_{q}\right)_{i+1 / 2, j+1 / 2}^{n+1 / 2}-\left(\zeta_{q}\right)_{i+1 / 2, j+1 / 2}^{n}}{\tau^{n+1 / 2} / 2}+\left(u_{q}\right)_{i+1 / 2, j+1 / 2} \frac{\left(\zeta_{q}\right)_{i+1, j+1 / 2}^{n}-\left(\zeta_{q}\right)_{i, j+1 / 2}^{n}}{h}
$$


Here, $\left(u_{q}\right)_{i+1 / 2, j+1 / 2}=\frac{1}{2}\left(\left(u_{q}\right)_{i+1, j+1 / 2}^{n+1}+\left(u_{q}\right)_{i, j+1 / 2}^{n+1}\right)$ in the domain interior, and $\left(u_{q}\right)_{i+1 / 2, j+1 / 2}=0$ on the boundary. In (39), the first-order accuracy in time can not affect the overall order of accuracy of the algorithm, because approximation (39) is used only in the non-differential constraint (36).

Once the flux variables are updated at the new time step, the new values of the centre-cell conservation variables are computed at the corrector step:

$$
\begin{aligned}
& \frac{\left(\zeta_{q}\right)_{i+1 / 2, j+1 / 2}^{n+1}-\left(\zeta_{q}\right)_{i+1 / 2, j+1 / 2}^{n+1 / 2}}{\tau^{n+1 / 2} / 2}+\frac{\left(u_{q}\right)_{i+1, j+1 / 2}^{n+1}\left(\zeta_{q}\right)_{i+1, j+1 / 2}^{n+1}-\left(u_{q}\right)_{i, j+1 / 2}^{n+1}\left(\zeta_{q}\right)_{i, j+1 / 2}^{n+1}}{h}+ \\
& \quad+\frac{\left(v_{q}\right)_{i+1 / 2, j+1}^{n+1}\left(\zeta_{q}\right)_{i+1 / 2, j+1}^{n+1}-\left(v_{q}\right)_{i+1 / 2, j}^{n+1}\left(\zeta_{q}\right)_{i+1 / 2, j}^{n+1}}{h}=\left(F_{q}\right)_{i+1 / 2, j+1 / 2}^{n+1 / 2}, \quad q=\overline{1 . .3} .
\end{aligned}
$$

For well-resolved solution regions, where the corrections (34) and (35) remain inactive, the CABARET scheme (27) - (40) approximates the conservation laws (19) to the second order of accuracy in both space and time, provided that the approximation of the full source term, $\left(F_{q}\right)_{i+1 / 2, j+1 / 2}^{n+1 / 2}$, is second-order accurate.

In our algorithm, the full source term is separated into the combined wind and viscous component and into the $\beta$-term component:

$$
\left(F_{q}\right)_{i+1 / 2, j+1 / 2}^{n+1 / 2}=\left(F_{q}^{\text {visc }+ \text { wind }}\right)_{i+1 / 2, j+1 / 2}^{n+1 / 2}+\left(F_{q}^{\text {Coriolis }}\right)_{i+1 / 2, j+1 / 2}^{n+1 / 2}
$$

We find that in the ocean simulations the $\beta$-term component is numerically stiff, and, therefore, it can excite spurious basin-scale barotropic modes. In order to avoid this problem, a separate second order accurate predictor-corrector Adams-type scheme is used in order to increase the stability of the approximation in time:

$$
\left(F_{q}^{\text {Coriolis }}\right)_{i+1 / 2, j+1 / 2}^{n+1 / 2}=(1+\delta)\left(R_{q}\right)_{i+1 / 2, j+1 / 2}^{n}-\delta\left(R_{q}\right)_{i+1 / 2, j+1 / 2}^{n-1}, \quad \delta=\frac{\tau^{n+1 / 2}}{2 \tau^{n-1 / 2}}
$$


where the residual is

$$
\left(R_{q}\right)_{i+1 / 2, j+1 / 2}^{n}=-\frac{1}{2} \beta\left(\left(v_{q}\right)_{i+1 / 2, j+1}^{n}+\left(v_{q}\right)_{i+1 / 2, j}^{n}\right)
$$

In our numerical algorithm, the $\beta$-term component (42) is added to the equations at the predictor time step (27).

Since, unlike in the Arakawa method, in the absence of viscous forces our method is equivalent to the conservation law of PV anomaly plus the $\beta$-term, rather than to the conservation law of PV itself, $\iint\left(\zeta_{q}+\beta \cdot y\right) d x d y=$ const,$\quad q=\overline{1 . .3}$, we need to make sure that our scheme is compatible with the discrete PV conservation law. This is indeed the case, since (43) is equivalent to the following conservative approximation of the $\beta$-term component:

$$
\begin{aligned}
& \left(R_{q}\right)_{i+1 / 2, j+1 / 2}^{n}=\text { Flux }_{j+1}-\text { Flux }_{j}+\text { Flux }_{i+1}-\text { Flux }_{i}+O(\operatorname{div}), \\
& \text { Flux }_{j}=-\beta y_{j}\left(v_{q}\right)_{i+1 / 2, j}^{n} / h, \text { Flux }_{i}=-\beta y_{j+1 / 2}\left(u_{q}\right)_{i, j+1 / 2}^{n} / h \\
& y_{j+1 / 2}=\frac{1}{2}\left(y_{j+1}+y_{j}\right),
\end{aligned}
$$

and $O($ div $)=\beta y_{j+1 / 2}\left[\frac{\left(u_{q}\right)_{i+1, j+1 / 2}^{n}-\left(u_{q}\right)_{i, j+1 / 2}^{n}}{h}+\frac{\left(v_{q}\right)_{i+1 / 2, j+1}^{n}-\left(v_{q}\right)_{i+1 / 2, j}^{n}}{h}\right]=0$, because the velocity field obtained from (29) and (30) is divergence-free. We have checked that the velocity divergence term is indeed of the order of round off error in all our calculations.

In contrast to the $\beta$-term component, the other component of the source term is numerically benign, and, therefore, it is approximated at the mid-time level by the second-order differences of both the velocity streamfunction and its Laplacian. The corresponding term is added to the equations after the elliptic problem (29) is solved, and after the velocity streamfunction at the mid-time level is updated: 


$$
\left(F_{q}^{\text {visc }+ \text { wind }}\right)_{i+1 / 2, j+1 / 2}^{n+1 / 2}=\delta_{1 q} \cdot f_{\text {wind }}+\delta_{3 q} \cdot \mu_{b o t}\left(\nabla^{2} \psi_{q}\right)_{i+1 / 2, j+1 / 2}^{n+1 / 2}+\mu_{\text {vol }}\left(\nabla^{2} D_{q}\right)_{i+1 / 2, j+1 / 2}^{n+1 / 2} .
$$

Here, the Laplacians are evaluated directly from the solution of elliptic problems at the mid-time level:

$$
\begin{aligned}
& \left(\nabla^{2} \psi_{1}\right)_{i+1 / 2, j+1 / 2}^{n+1 / 2}=\left(\zeta_{1}\right)_{i+1 / 2, j+1 / 2}^{n+1 / 2}+S_{1}\left(\left(\psi_{1}\right)_{i+1 / 2, j+1 / 2}^{n+1 / 2}-\left(\psi_{2}\right)_{i+1 / 2, j+1 / 2}^{n+1 / 2}\right), \\
& \left(\nabla^{2} \psi_{2}\right)_{i+1 / 2, j+1 / 2}^{n+1 / 2}=\left(\zeta_{2}\right)_{i+1 / 2, j+j+1 / 2}^{n+1 / 2}+s_{21}\left(\left(\psi_{2}\right)_{i+1 / 2, j+1 / 2}^{n+1 / 2}-\left(\psi_{1}\right)_{i+1 / 2, j+1 / 2}^{n+1 / 2}\right)+s_{22}\left(\left(\psi_{2}\right)_{i+1 / 2, j+1 / 2}^{n+1 / 2}-\left(\psi_{3}\right)_{i+1 / 2, j+1 / 2}^{n+1 / 2}\right), \\
& \left(\nabla^{2} \psi_{3}\right)_{i+1 / 2, j+1 / 2}^{n+1 / 2}=\left(\zeta_{3}\right)_{i+1 / 2, j+1 / 2}^{n+1 / 2}+S_{3}\left(\left(\psi_{3}\right)_{i+1 / 2, j+1 / 2}^{n+1 / 2}-\left(\psi_{2}\right)_{i+1 / 2, j+1 / 2}^{n+1 / 2}\right) .
\end{aligned}
$$

In order to untangle the variables in the elliptical problem (45), the vertical normal-mode variables are employed. They allow to diagonalise the elliptic problem into the three independent Helmholtz problems (for the individual vertical modes), that are solved with the combined Fast Fourier Transform (FFT) and cyclic reduction methods.

\section{Ocean modelling results}

In this section we discuss the ocean circulation solutions obtained with the CABARET algorithm and compare the results with those obtained by using the conventional second-order Arakawa method. The Arakawa method is based on the CL advection scheme and Robert-Asselin filtering in time (with the relaxation constant equal to 0.1 ). We have actually checked that the results reported in this section for the Arakawa method are virtually insensitive to the relaxation constant in the range 0.01-0.3. Both methods are implemented in the same quasiqeostrophic ocean modelling code that (with the Arakawa method) was previously used in double-gyre simulations (e.g., Berloff et al., 2007). We also checked that, in comparison with the conventional Arakawa method, the CABARET method (24)-(42) is about 1.2-2 times slower. However, this is a small drawback relative to the advantage of the greatly increased accuracy of the model. 


\subsection{Moderate Reynolds numbers}

First, we compare the CABARET method with the conventional second-order method and with a lowdispersion first-order upwind method. We use $\mu_{\text {eddy }}=50 \mathrm{~m}^{2} \mathrm{~s}^{-1}$ that corresponds to a moderate-Re flow regime, $R e=3200$. We use 4 uniform Cartesian grids with $129 \times 129,257 \times 257,513 \times 513$, and $1025 \times$ 1025 nodes. For the moderate-Re case the solution convergence is confirmed against the finest grid, 2059x2059 simulation, which is almost indistinguishable from the fine 1025-grid solution (e.g. the difference of maximum r.m.s. fluctuations of the eastward jet is less than $0.1 \%$ ). Each simulation starts from the state of rest, and each physical run time is $10^{4}$ days, which corresponds to about $0.5-1$ million time steps on the fine, $1025^{2}$ grid. The spin-up time, during which all solutions reach statistically stationary states, is 8000 days. The data for further processing are stored for the remaining physical time of 2000 days, which is long relative to most of the characteristic time scales of the mesoscale eddies. For the purpose of the grid convergence study, we consider the time averages of the solutions and their r.m.s. fluctuations. At the moderate $\mathrm{Re}=3200$, the Arakawa scheme begins to converge starting from the 513-grid (Fig. 8,9). On the 257-grid, the western boundary current eastward jet (EJ) separates below the correct latitude (as given by the converged finest-grid solution). We suggest that this spurious behaviour of the EJ is an artifact of the numerical dissipation and dispersion errors that can be significantly corrected with the CABARET algorithm. Overall, correct modelling of the EJ is one of the key targets that we seek to achieve on relatively coarse grids.

The convergence of the CABARET and Arakawa methods have been checked in 3 error norms and by the location of the separation point of the jet on the EJ (table 2). The first 2 errors correspond to the standard maximum norm of the time-averaged upper-ocean streamfunction solution and to that of the r.m.s. fluctuations of the upper-ocean streamfunction solution, respectively. The third error corresponds to the absolute value of the relative error in predicting the maximum of the r.m.s. 
fluctuations of the upper-ocean streamfunction solution that characterises the strength of the EJ. As seen from the table, the CABARET scheme shows significantly smaller errors and faster convergence rate for all error norms in comparison to the Arakawa scheme, which for Max $\left\|\varepsilon_{\psi_{1}}\right\|$ norm even fails to converge. The CABARET convergence rate is approximately linear in the maximum norms. For both methods, the relative error of the maximum r.m.s. jet fluctuations decays faster than the quadratic rate, with the CABARET method being still superior. The convergence of the EJ separation point is in table 3, and it also shows a superiority of the CABARET solution.

Table 2. Error convergence for the moderate-Re case

\begin{tabular}{|l|l|l|l|l|l|l|}
\hline Grid & CABARET & Arakawa & CABARET & Arakawa & CABARET & Arakawa \\
& $\operatorname{Max}\left\|\varepsilon_{\psi_{1}}\right\|$ & $\operatorname{Max}\left\|\varepsilon_{\psi_{1}}\right\|$ & $\operatorname{Max}\left\|\varepsilon_{r . m . s .\left(\psi_{1}\right)}\right\|$ & $\operatorname{Max}\left\|\varepsilon_{r . m . s .\left(\psi_{1}\right)}\right\|$ & $\varepsilon \| \max \left(\right.$ r.m.s. $\left.\left(\psi_{1}\right)\right) \|$ & $\varepsilon \| \max \left(\right.$ r.m.s. $\left.\left(\psi_{1}\right)\right) \|$ \\
\hline 129 & $\mathbf{1 4 2 . 2 2}$ & 138.48 & $\mathbf{9 9 . 3 9}$ & 104.25 & $\mathbf{0 . 4 7}$ & 0.315 \\
\hline 257 & $\mathbf{1 3 2 . 6 6}$ & 189.07 & $\mathbf{5 6 . 5 8}$ & 86.87 & $\mathbf{0 . 0 7 1}$ & 0.098 \\
\hline 513 & $\mathbf{5 0 . 9 8}$ & 199.44 & $\mathbf{3 4 . 4 2}$ & 71.29 & $\mathbf{0 . 0 0 3}$ & 0.012 \\
\hline
\end{tabular}

Table 3. Convergence of the location of the separation point, $y / L$.

\begin{tabular}{|l|l|l|l|}
\hline Grid & 257 & 513 & 1025 \\
\hline Arakawa & 0.4 & 0.5 & 0.55 \\
\hline CABARET & $\mathbf{0 . 5}$ & $\mathbf{0 . 5 5}$ & $\mathbf{0 . 5 5}$ \\
\hline
\end{tabular}

The confidence interval for the error in separation point location, which was estimated by graphical data post-processing in Tecplot, is about \pm 0.01 . 


\subsection{High Reynolds numbers}

The advantages of the CABARET method over the conventional second-order methods are more pronounced at high Re. To demonstrate this, we computed the double-gyre problem for a large $\operatorname{Re} \approx 13300\left(\mu_{e d d y}=12 m^{2} s^{-1}\right)$.

With our grid resolutions, and, unlike in the moderate-Re case (Section 4.1), there is no evidence of the solution convergence for either of the schemes except for the jet separation point location on the western boundary (Table 4).

Fig.10 and Fig.11 compare time averaged and r.m.s. fluctuations of the upper-ocean velocity streamfunction for the coarse 257-grid Arakawa and CABARET solutions with that of the fine 1025grid Arakawa solution. We find that in contrast to the coarse-grid Arakawa solution the other two solutions are very similar. This similarity is further examined by plotting typical instantaneous snapshots of the upper-ocean streamfunction and PV anomaly for the same solutions (Fig.12,13). In all figures the same contour levels of the streamfunction and PV anomaly are plotted. While the 257-grid Arakawa solution is strongly contaminated by spurious short-wave oscillations which completely obliterate the EJ structure, the 257-grid CABARET solution is remarkably close to the reference fine 1025-grid solution.

To further validate this qualitative picture, we have checked that the underprediction of the maximum r.m.s. fluctuations of the EJ of the 257-grid Arakawa solution, in comparison to the reference 1025 solution, is $65 \%$, thus indicating a total failure of the solution. In contrast to this, the CABARET solution on the 257 grid results in only $19 \%$ error in the maximum r.m.s. fluctuations of the EJ. We find this qualitative agreement with the 1025-grid solution remarkable, given that computation with the coarse 257-grid CABARET is more than 30 times faster than that with the fine 1025-grid Arakawa scheme. 
Table 4. Location of the separation point, $y / L$

\begin{tabular}{|l|l|l|l|}
\hline Grid & 257 & 513 & 1025 \\
\hline Arakawa & N/A & 0.3 & 0.5 \\
\hline CABARET & $\mathbf{0 . 5}$ & $\mathbf{0 . 5 5}$ & $\mathbf{0 . 5}$ \\
\hline
\end{tabular}

See the footnotes to Table 3

\section{Conclusions and Discussion}

The novel Compact Accurately Boundary Adjusting high-REsolution Technique (CABARET) works remarkably well for the eddy-resolving quasigeostrophic double-gyre ocean circulation problem. In comparison with the conventional second-order method, it allows to increase Reynolds number (Re) of the simulations by an order of magnitude - this opens exciting perspectives for exploring more realistic flow regimes.

The CABARET approach is based on several important ideas, which have been circulating in the computational physics community:

(i) Fully discrete/Lagrangian property: optimal approximation of the entire material derivative rather than optimisation of the time and space discretisations separately, as in the standard Eulerian schemes;

(ii) Low dispersion and no-dissipation error of the underlining finite-difference scheme;

(iii) Non-oscillatory property: enforcing the maximum principle on the solution as the means for efficient treatment of the underresolved scales;

(iv) Ease of implementation for complicated boundary conditions and non-uniform grids due to the compactness of stencil in space and time

(v) Reduced CPU cost due to the small computational stencil 
The novelty of our approach is in achieving good dispersion and dissipation properties of the underlying advection scheme that are crucial in large-Re eddy-resolving simulations. We deal with these properties in the situation when the regularising effect of the explicit eddy viscosity is small relative to the dissipation and dispersion errors of the scheme. In order to prove this point, we presented a systematic comparison of the new CABARET method with popular high-resolution methods such as MUSCL, PPM and WENO, for several linear-advection and gas dynamics problems. Finally, we contrasted the performance of the CABARET scheme with that of the standard second-order Arakawa method for the classical ocean circulation problem. In particular we show that the numerical dispersion and dissipation error of the latter can be responsible for largely incorrect eastward jet structure.

For the double-gyre circulation, the CABARET method allows one to obtain high-quality solutions on the grid that has half a point over the Munk lengthscale, $\left(\mu_{\text {eddy }} / \beta\right)^{1 / 3}$, which characterises the viscous western boundary layer. This is a very significant upgrade relative to the conventional scheme that requires 2-3 grid points over this lengthscale (e.g., Berloff et al., 2007).

In the current publication we haven't attempted to implement other high-resolution schemes, such as those quoted in Section 2, for the double-gyre problem and to compare them with the CABARET method. This is because of the complexity of implementation of the former near the boundaries (e.g., the western boundary treatment is a crucial part of the double-gyre solution), since many such methods have a larger computational stencil in comparison to the CABARET or Arakawa scheme.

Potential future directions include adaptation of the CABARET method for non-uniform grids, which can be achieved by capitalising on the remarkable compactness of its computational stencil, and extension of the method to the primitive equations for use in the comprehensive ocean general circulation models. 


\section{Acknowledgements}

Supports from the Royal Society of London and from the Mary Sears Visitor Grant are acknowledged by SK with gratitude. The work of VG was supported by the Russian Foundation for Basic Research (RFBR), grant 06-01-00819a. Funding for PB was provided by the NSF grant 0725796. Discussions with Alex Shchepetkin and Nikolay Dianskii are graciously appreciated.

\section{References}

1. Arakawa, A., Computational design for long-term numerical integration of the equations of fluid motion: Two-dimensional incompressible flow. Part I, J. Comput. Phys., 1 (1966), pp. 119-143.

2. Asselin, R. Frequency filter for time integrations. Mon. Wea. Rev., 1972, 100, pp. $487-490$.

3. Berloff, P., Hogg, A., and Dewar, W., 2007: The turbulent oscillator: A mechanism of lowfrequency variability of the wind-driven ocean gyres. J. Phys. Oceanogr., 37, pp. 2363-2386.

4. Bogey, C. and Bailly, C., A family of low dispersive and low dissipative explicit schemes for flow and noise computations. J. Comput. Physics, 194 (2004), pp. 194-214.

5. Boris, J.P., Book, D.L., and Hain, K., Flux-corrected transport: Generalization of the method, $J$. Comput. Phys, 31,(1975), 335-350.

6. Carpenter, R. L., Droegemeier, K. K., Woodward, P. R., and Hane, C. E., Application of the piecewise parabolic method (ppm) to meteorological modeling. Monthly Weather Review 118, 586-612, 1990.

7. Cockburn B and Shu CW: Runge-Kutta Discontinuous Galerkin Methods for ConvectionDominated Problems, Journal of Scientific Computing, 16(3): 173-261, Sept 2001. 
8. Colonius, T. and Lele, S.K., Computational aeroacoustics: progress on nonlinear problems of sound generation. Progress in Aerospace sciences 40 (2004), pp. 345-416.

9. Crowley, W.P.: 1968, Numerical Advection Experimens, Mon. Wea. Rev., 96, 1-12.

10. Donat, R., and Marquina, A. "Capturing Shock Reflections: An Improved Flux Formula", Journal of Computational Physics, 1996, V.25, pp.42-58.

11. Drikakis, D. 2003 Advances in turbulent flow computations using high-resolution methods, Prog. Aerosp. Sci. 39, pp. 405-424.

12. Dritschel, D.G., Polvani, L.M. and Mohebalhojeh, A.R.: The contour-advective semiLagrangian algorithm for the shallow water equations. Mon. Wea. Rev. 127(7), pp. 1551-1565 (1999).

13. Godunov, S.K.: 1959, A difference scheme for numerical computation of discontinuous solutions of equations of fluid dynamics, Math.Sb. 47(89), pp. 271-306.

14. Goloviznin, V.M. and Samarskii, A.A., Difference approximation of convective transport with spatial splitting of time derivative, Mathematical Modelling, 1998a, 10(1), pp. 86-100.

15. Goloviznin, V.M. and Samarskii, A.A., Some properties of the CABARET scheme, Mathematical Modelling, 1998b, 10(1), pp. 101-116

16. Goloviznin, V.M. and Karabasov, S.A., Non-linear correction of Cabaret scheme. Mathematical Modelling, 10(1998), 12, pp. 107-123.

17. Goloviznin, V.M., Karabasov, S.A. and Kobrinski, I.M. Balance-Characteristic Schemes with Staggered Conservative and Transport Variables. Math. Modelling Journal, V.15 (2003), N.9, pp.29-48.

18. Goloviznin, V.M., Semenov, V.N., Korortkin, I.A., and Karabasov, S.A., A novel computational method for modelling stochastic advection in heterogeneous media. Transport in Porous Media, Springer Netherlands, 2007, 66(3), pp. 439-456. 
19. Goloviznin, V.M., Balanced Characteristic Method for Systems of Hyperbolic Conservation Laws, Doklady Mathematics, 72(1), July-August 2005, pp. 619-623.

20. Grinstein, F.F., Margolin, L.G and Rider. W.J. 2007 Implicit large eddy simulation: computing turbulent dynamics, ch.5, pp. 195-220. Cambridge University Press, New York.

21. Hundsdorfer, W., Koren, B., van Loon M., and Verwer, J.G. A positive finite-difference advection scheme. J. Comput. Phys. 1995, 117, pp. 35-46.

22. Harten, A., Engqist, B., Osher, S., and Chakravarthy, S. Uniformly High Order Accurate Essentially Non-Oscillatiry Schemes III, J. Comput. Phys, 71, (1987), pp. 231-303

23. Hirsch C. Numerical Computation of Internal and External Flows. The Fundamentals of Computational Fluid Dynamics, $2^{\text {nd }}$ Edition, John Wiley\&Sons, Ltd 2007.

24. Holland, W., The role of mesoscale eddies in the general circulation of the ocean - Numerical experiments using a wind-driven quasigeostrophic model. J. Phys. Oceanogr., 1978, 8, pp. 363 $-392$.

25. Iserles, A., Generalized Leapfrog Methods, IMA Journal of Numerical Analysis, 6 (1986), 3, pp. 381-392.

26. Karabasov, S.A. and Goloviznin, V.M. "Digital Transport Approach for hyperbolic-type problems". 2004, Hyperbolic Problems: Theory, Numerics and Applications, part II, pp. 79-86. Yokohama Publishers: ISBN 4946552-21-9.

27. Karabasov, S.A. and Goloviznin, V.M. New Efficient High-Resolution Method for Nonlinear Problems in Aeroacoustics, AIAA Journal, 2007, vol. 45, no. 12, pp. $2861-2871$

28. Kim, S. High-order upwind leapfrog methods for multidimensional acoustic equations, Int J. Numer. Mech. Fluids, 44 (2004), pp. 505-523. 
29. Kravchenko, A. and Moin, P. 1997. On the effect of numerical errors in Large Eddy Simulations of turbulent flows, J.Comput. Physics, 131, pp. $310-322$.

30. van Leer, B. Towards the Ultimate Conservative Difference Scheme, V. A Second Order Sequel to Godunov's Method, J. Comput. Phys. (1979) 32: 101-136.

31. Lele, S.K., Compact finite-difference scheme with spectral-like resolution, J.Comput. Physics, 103 (1992), 16-42.

32. LeVeque, R.J. "Finite volume methods for hyperbolic problems", Cambridge University Press, 2002

33. Margolin, L.G. and Shashkov, M. 2004. Remapping, recovery and repair on a staggered grid. Comput. Methods Appl. Mech. Engng, 193, pp. 4139 - 4155.

34. Margolin, L.G. 1997 Introduction to "An arbitrary Lagrangian-Eulerian computing method for all flow speeds”, J.Comput. Physics, 135, pp. 198 - 202.

35. McWilliams, J., 1977: A note on a consistent quasigeostrophic model in a multiply connected domain. Dyn. Atmos. Oceans, 1, pp. 427-441.

36. Mohebalhojeh, A.R. and Dritschel, D.G.: Contour-advective semi-Lagrangian algorithms for manylayer primitive equation models. Quart. J. Roy. Meteorol. Soc. 130, pp. 347-364 (2004).

37. von Neumann, J. and Richtmyer, R. D. A method for the numerical calculation of hydrodynamic shocks, 1950. J. Appl. Physics, 1950, 21, pp. 232-237.

38. Ostapenko V.V., On the monotonocity of the balanced-characteristic scheme, Mathematical Modelling Journ, 2009, Vol. 21 (7), pp. 29-42.

39. Pietrzak, J.. The use of TVD limiters for forward-in-time upstream-biased advection schemes in ocean modeling. Monthly Weather Review 126, 812-830, 1998.

40. Pope, S. Turbulent Flows, 2000, Cambridge University Press, Cambridge.

41. Roache, P.J. Computational Fluid Dynamics, Hermosa, Albuquerque, 1982. 
42. Robert, A.J. The integration of a low-order spectral form of the primitive meteorological equations. J. Met. Soc. Japan. 1966, 44 (5), pp.237 - 245.

43. Roe, P.L. Linear bicharacteristic schemes without dissipation, SISC, 19, pp. 1405-1427, 1998

44. Rozhdestvensky, B.L. and Yanenko, N.N. Systems of quasilinear equations, Moscow, Nauka, 1978.

45. Shchepetkin, A.F. and McWilliams, J. C. 1998, Quasi-monotone Advection Schemes Based on Explicit Locally Adaptive Dissipation; Monthly Weather Review, 126 , pp. 1541-1580.

46. Shu, C.W. and Osher, S., Efficient implementation of essentially non-oscillatory shockcapturing schemes, J.Comp. Phys., 77 (1988), 439-471.

47. Starr, V., 1968: Physics of negative viscosity phenomena. McGraw-Hill, New York, 256 pp.

48. Tam, C.K.W. and Webb. J.C., Dispersion-relation-preserving finite difference schemes for computational acoustics, J.Comput. Physics, 107 (1993), 262-281.

49. Tabeling, P. "Two-dimensional turbulence: a physicist approach”, Physics Report, 362(2002), $1-62$.

50. Tran, Q.H. and Scheurer, B. "High-Order Monotonicity-Preserving Compact Schemes for Linear Scalar Advection on 2-D Irregular Meshes”, J. Comp. Phys. 2002, Vol. 175, Issue 2, pp. $454-486$.

51. Vallis, G.K. Atmospheric and ocean fluid dynamics, Cambridge University Press, 2006

52. Woodward, P. and Colella, P., The numerical simulation of two-dimensional fluid flow with strong shocks, 1984, J. Comp. Phys., 54, 115-173.

53. Zhou, Y. C. and Wei, G. W. High resolution conjugate filters for the simulation of flows. J.Comput.Physics, Volume 189, Issue 1, 20 July 2003, pp 159-179. 


\section{List of Figures}

Figure 1. Assumed data structure for the CABARET scheme for 1 dimension in space plus time. Solid circles denote conservation variables, open circles denote flux variables.

Figure 2. 1-D linear advection test, (a) - with a rectangular initial profile, (b) - with a half-sine-wave initial profile after 250 time steps on a uniform grid with $\mathrm{h}=1$ under $\mathrm{CFL}=0.45$; squares denote the solution of CABARET scheme, circles denote the solution of the second-order Van Leer scheme with TVD MinMod limiter, triangles denote the solution of the same scheme with TVD Superbee limiter.

Figure 3. Phase errors of several spatial finite-difference schemes and of the CABARET scheme at different CFL numbers $v s$ grid resolution (number of grid points per wavelength, $N_{\lambda}=\pi /(k \cdot h)$ ). E2, E4, E6 denote standard central differences of the second, fourth and sixth-order, respectively, DRP denotes the fourth order Dispersion Relation Preserving scheme (Tam and Webb, 1993); and LUI stands for a sixth order pentadiagonal compact scheme of Pade-type (Colonius and Lele, 2004). Results for all central schemes correspond to infinitesimally small Courant number, which corresponds to the highest accuracy for most of the central schemes, and CABARETx stands for the CABARET scheme at Courant number $\mathrm{CFL}=\mathrm{x}$.

Figure 4. Numerical group speeds of several central finite-difference schemes and of the CABARET scheme at different Courant numbers as functions of the grid resolution. The notations are the same as in Fig.3.

Figure 5. Assumed data structure for the CABARET scheme for 2 dimensions in space plus time. Notations are the same as in Fig.1. 
Figure 6. Solid body rotation test: cone profile after 1 full revolution on Cartesian grid with resolution of 20 grid cells per cone diameter.

Figure 7. Density solution profile for the Shu-Osher test for $\mathrm{t}=1.872$.

Figure 8. Convergence of the Arakawa and CABARET methods for the moderate-Re case. Timeaveraged, upper-ocean velocity streamfunction solutions: (a) 257 x 257 grid, (b) 513 x 513 grid, and (c) $1025 \times 1025$ grid.

Figure 9. Convergence of the Arakawa and CABARET methods for the moderate-Re case. R.m.s. fluctuation of upper-ocean velocity streamfunction solutions: (a) 257 x 257 grid, (b) 513 x 513 grid, and (c) 1025 x 1025 grid.

Figure 10. Comparative behaviour of solutions for the high-Re case. Time-averaged, upper-ocean velocity streamfunction solutions: (a) Arakawa method on 257 x 257 grid, (b) CABARET method on 257 x 257 grid, and (c) Arakawa method on 1025 x 1025 grid.

Figure 11. Comparative behaviour of solutions for the high-Re case. R.m.s. fluctuation of upper-ocean velocity streamfunction solutions: (a) Arakawa method on 257 x 257 grid, (b) CABARET method on 257 x 257 grid, and (c) Arakawa method on 1025 x 1025 grid.

Figure 12. Comparative behaviour of solutions for the high-Re case. Typical instantaneous upper-ocean velocity streamfunction: (a) Arakawa method on 257 × 257 grid, (b) CABARET method on 257 x 257 grid, and (c) Arakawa method on 1025 x 1025 grid.

Figure 13. Comparative behaviour of solutions for the high-Re case. Typical instantaneous upper-ocean PV anomaly: (a) Arakawa method on 257 x 257 grid, (b) CABARET method on 257 x 257 grid, and (c) Arakawa method on 1025 x 1025 grid. 


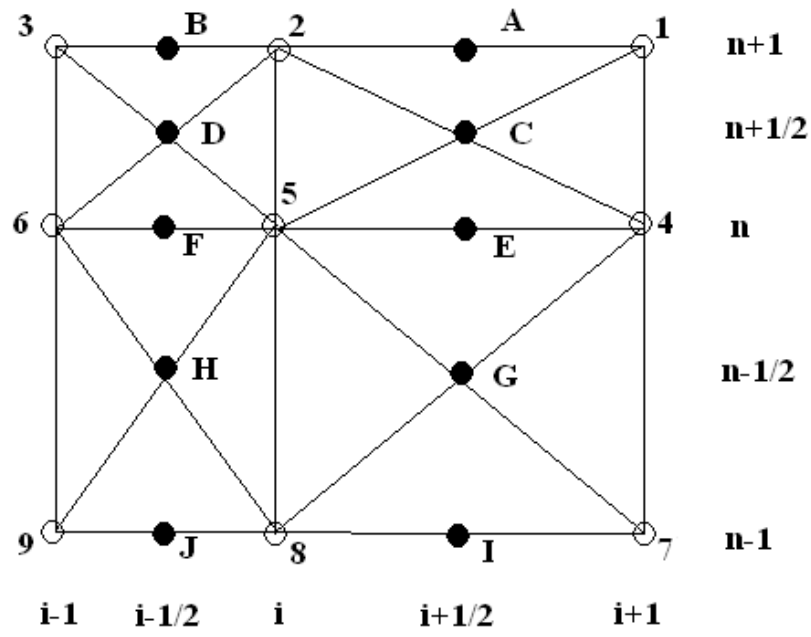

Figure 1. Assumed data structure for the CABARET scheme for 1 dimension in space plus time. Solid circles denote conservation variables, open circles denote flux variables. 
(a)

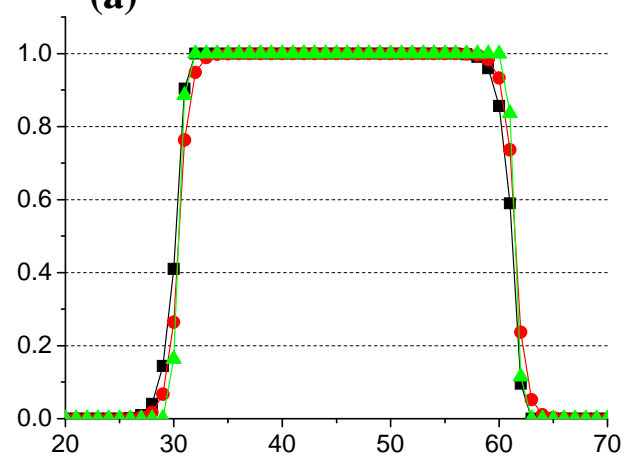

(b)

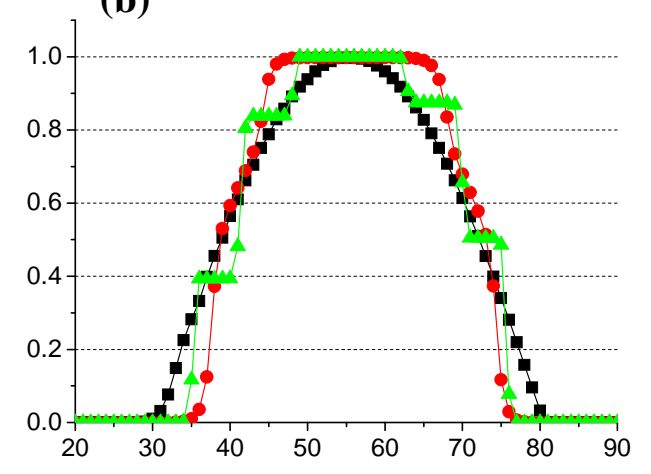

Figure 2. 1-D linear advection test, (a) - with a rectangular initial profile, (b) - with a half-sine-wave initial profile after 250 time steps on a uniform grid with $\mathrm{h}=1$ under $\mathrm{CFL}=0.45$; squares denote the solution of CABARET scheme, circles denote the solution of the second-order Van Leer scheme with TVD MinMod limiter, triangles denote the solution of the same scheme with TVD Superbee limiter. 


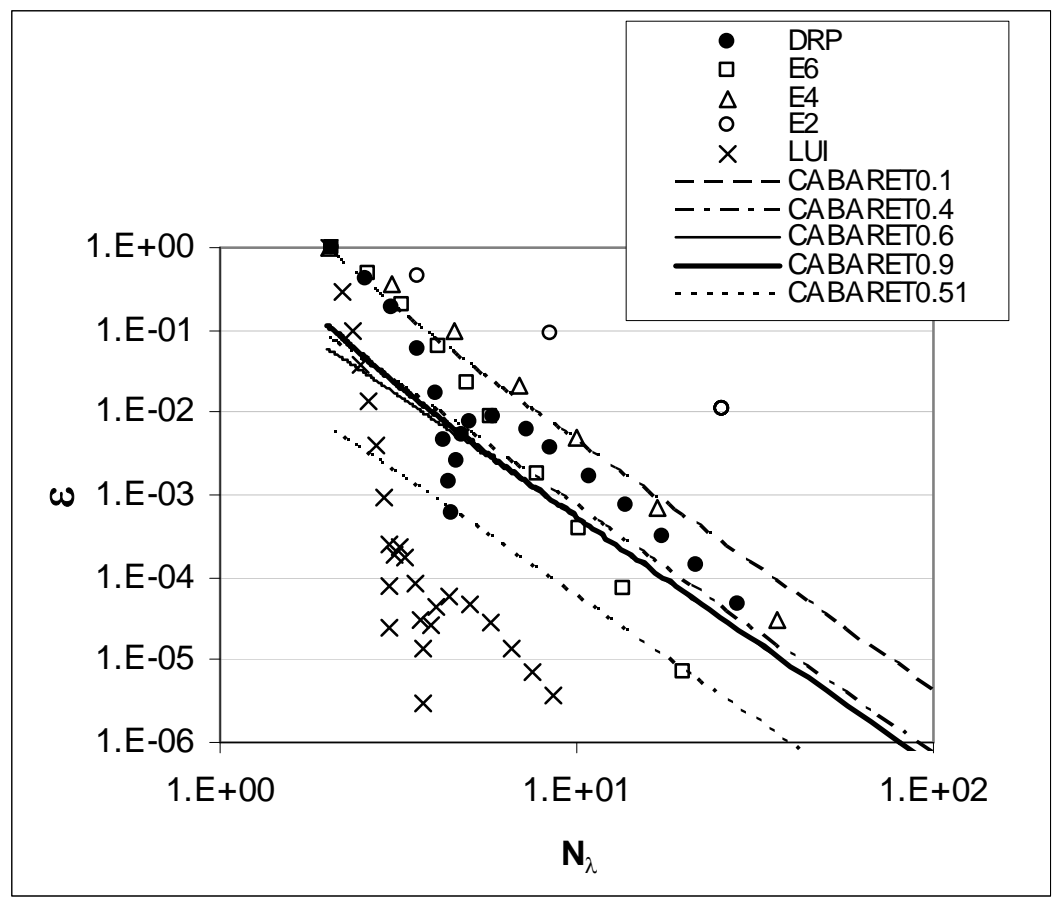

Figure 3. Phase errors of several spatial finite-difference schemes and of the CABARET scheme at different CFL numbers $v s$ grid resolution (number of grid points per wavelength, $N_{\lambda}=\pi /(k \cdot h)$ ). E2, E4, E6 denote standard central differences of the second, fourth and sixth-order, respectively, DRP denotes the fourth order Dispersion Relation Preserving scheme (Tam and Webb, 1993); and LUI stands for a sixth order pentadiagonal compact scheme of Pade-type (Colonius and Lele, 2004). Results for all central schemes correspond to infinitesimally small Courant number, which corresponds to the highest accuracy for most of the central schemes, and CABARETx stands for the CABARET scheme at Courant number $\mathrm{CFL}=\mathrm{x}$. 


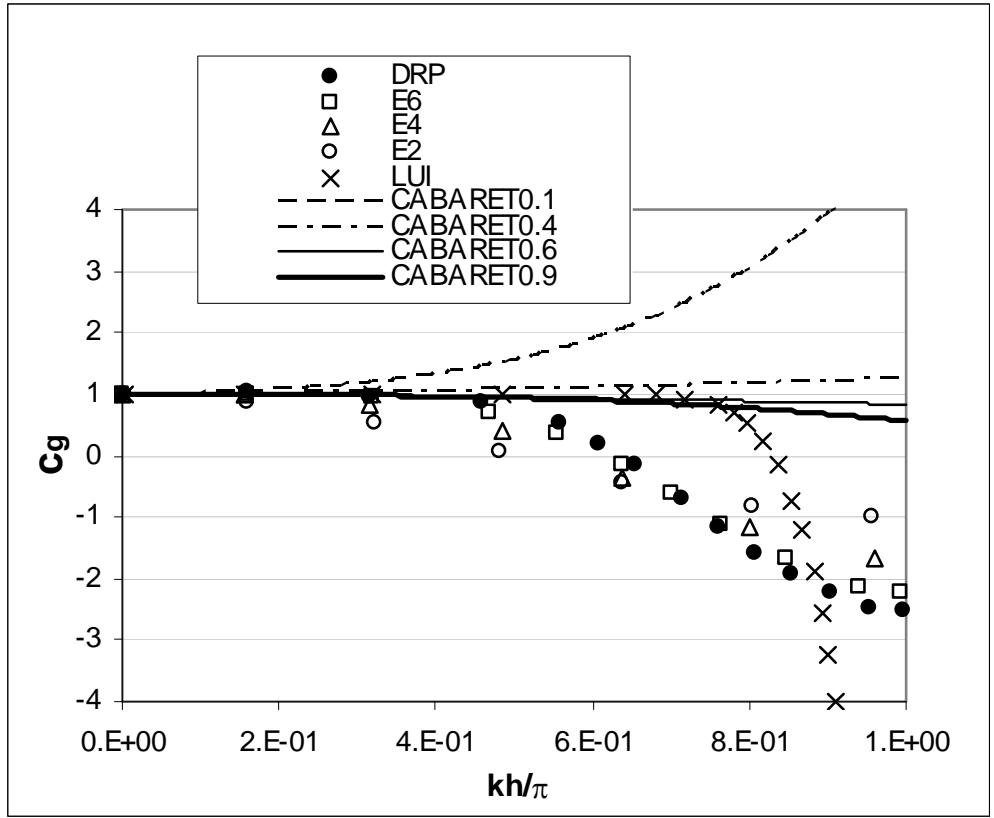

Figure 4. Numerical group speeds of several central finite-difference schemes and of the CABARET scheme at different Courant numbers as functions of the grid resolution. The notations are the same as in Fig.3. 


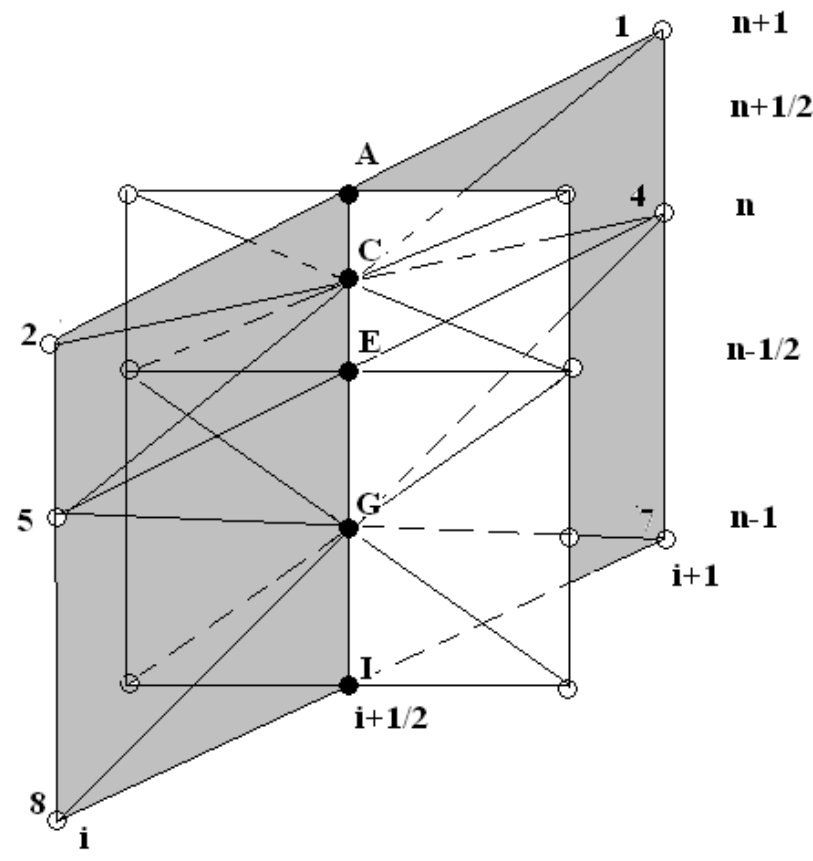

Figure 5. Assumed data structure for the CABARET scheme for 2 dimensions in space plus time.

Notations are the same as in Fig.1. 


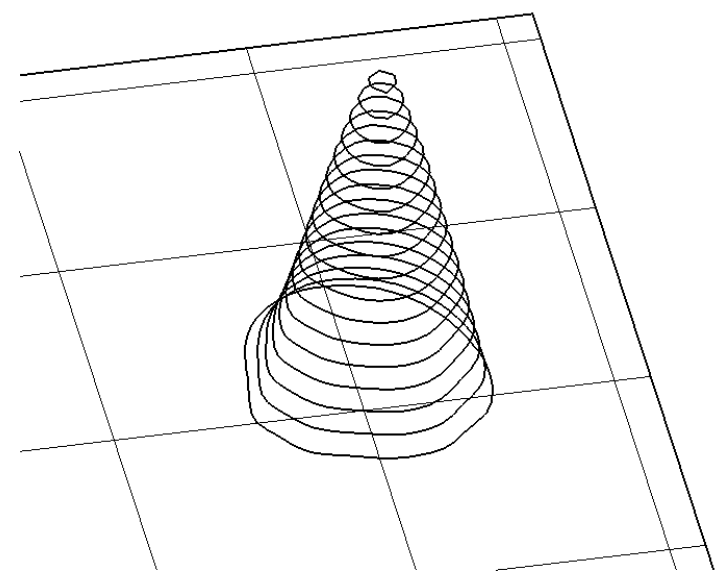

Figure 6. Solid body rotation test: cone profile after 1 full revolution on the Cartesian grid of resolution 20 grid cells per cone diameter. 


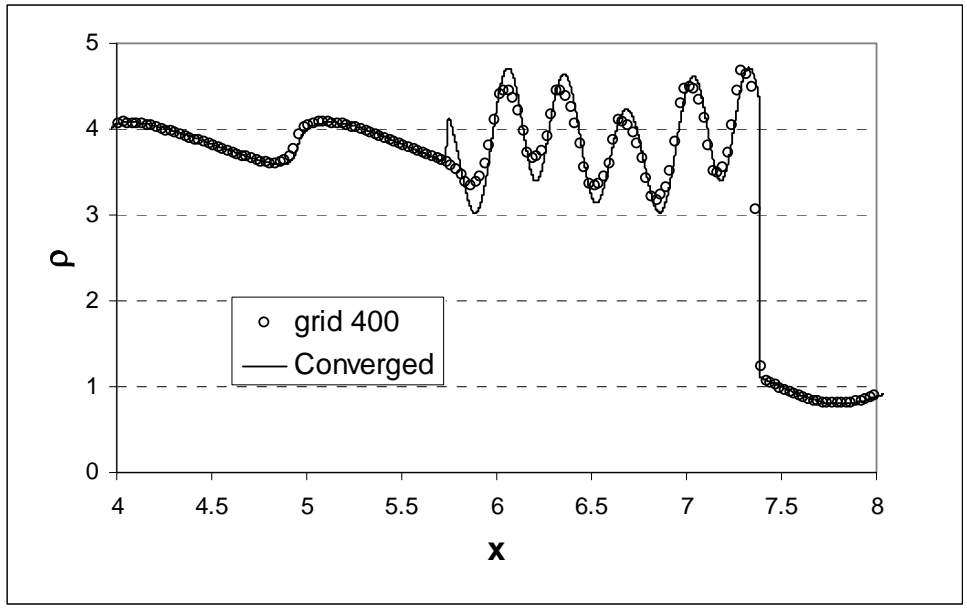

Figure 7. Density solution profile for the Shu-Osher test for $\mathrm{t}=1.872$. 
Arakawa. 257

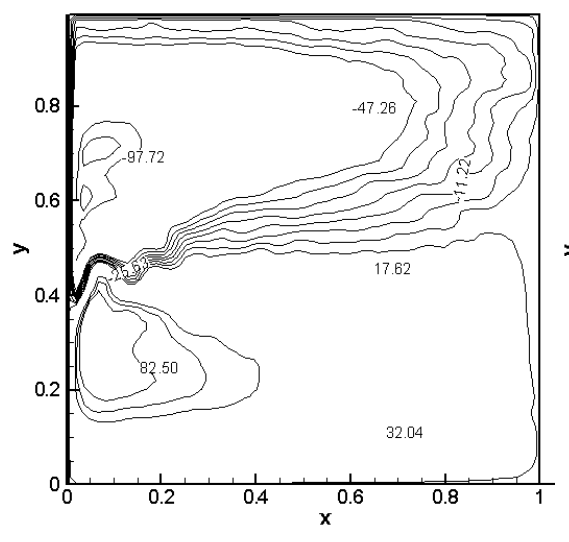

CABARET, 257

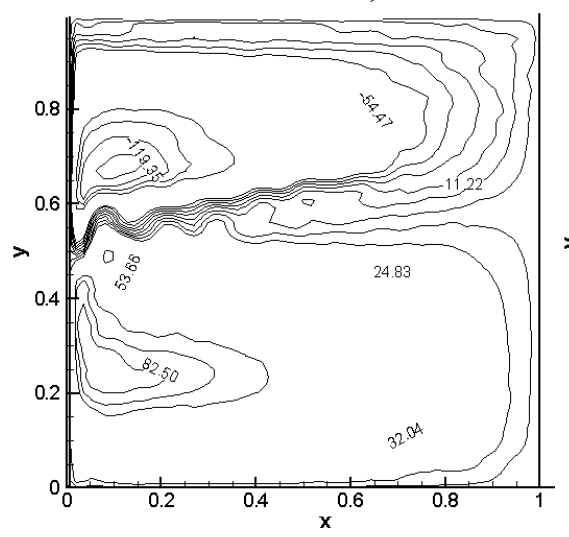

(a)
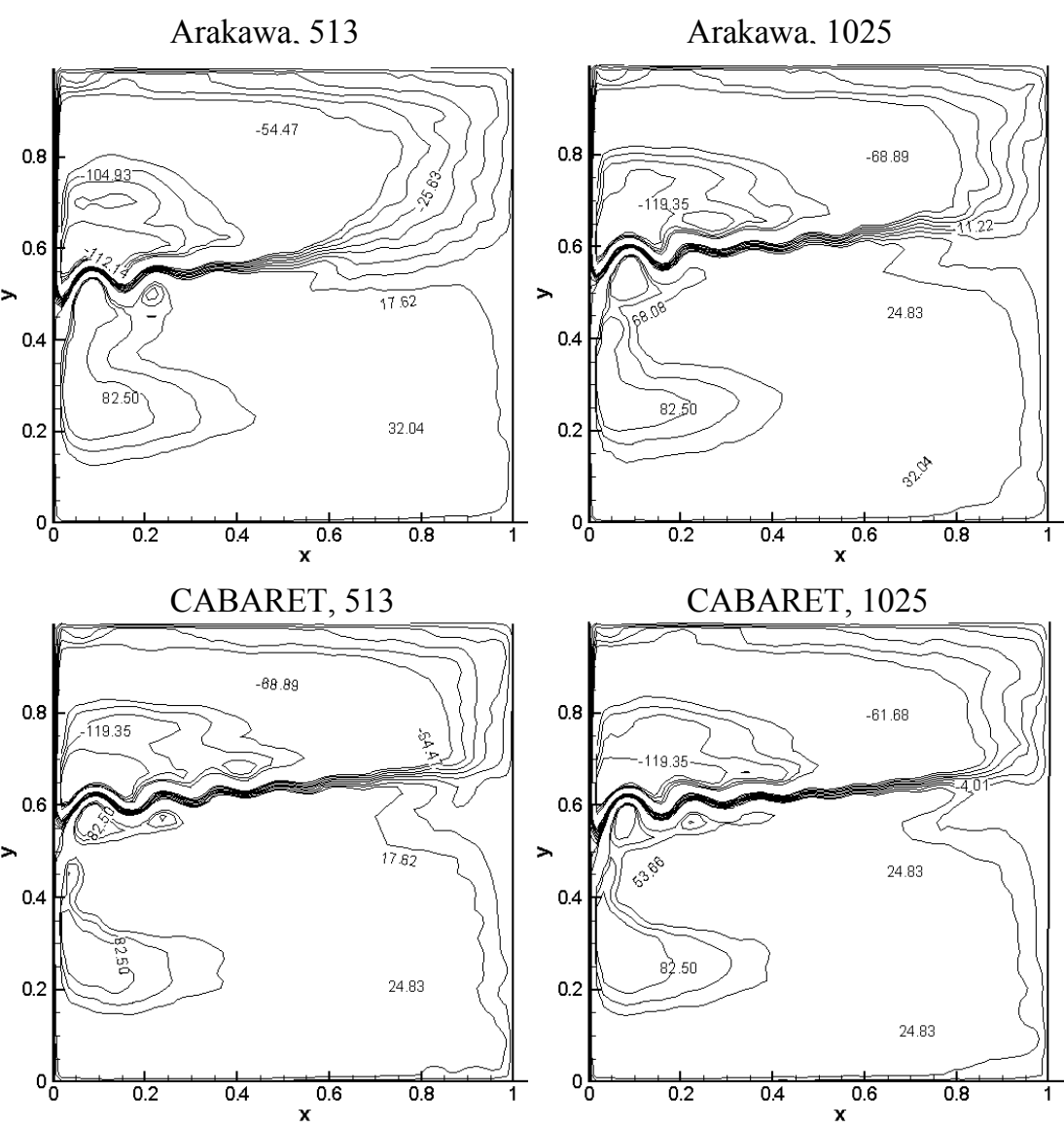

(b)

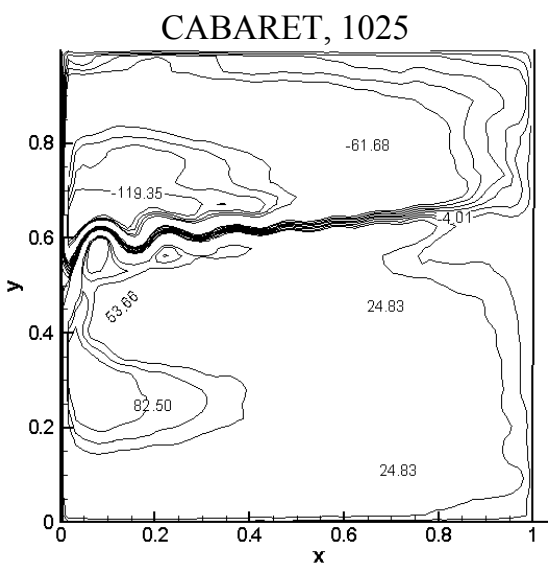

(c)

Figure 8. Convergence of the Arakawa and CABARET methods for the moderate-Re case. Timeaveraged, upper-ocean velocity streamfunction solutions: (a) 257 × 257 grid, (b) 513 × 513 grid, and (c) 1025 x 1025 grid. 
Arakawa. 257
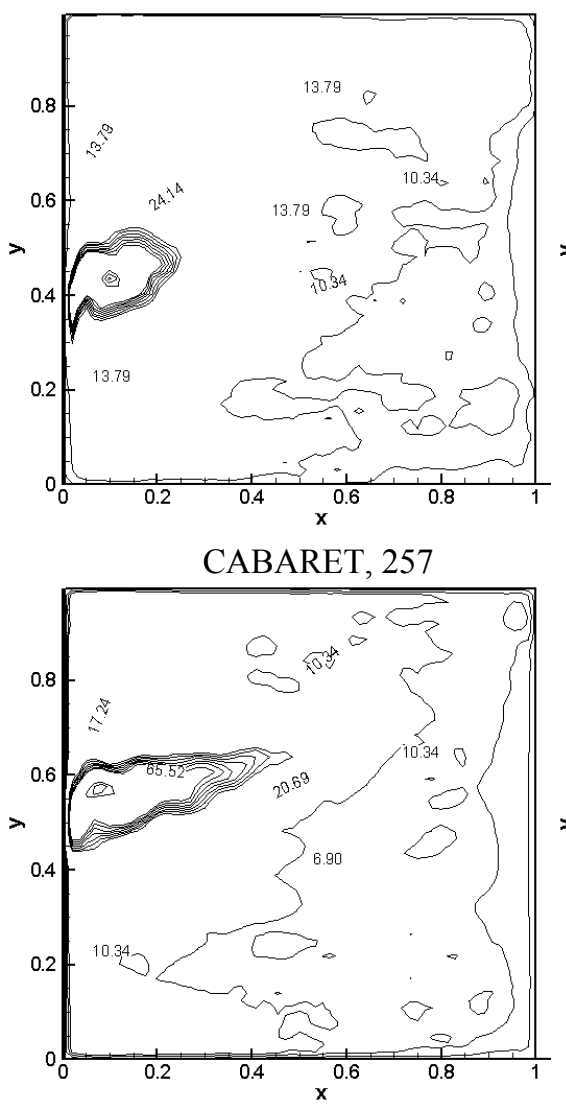

(a)
Arakawa. 513

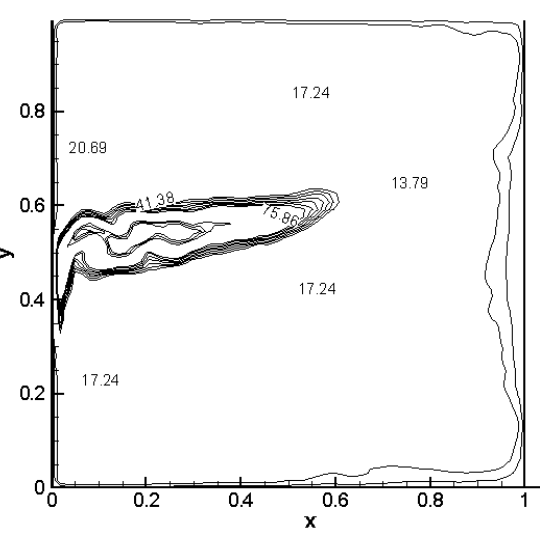

CABARET, 513

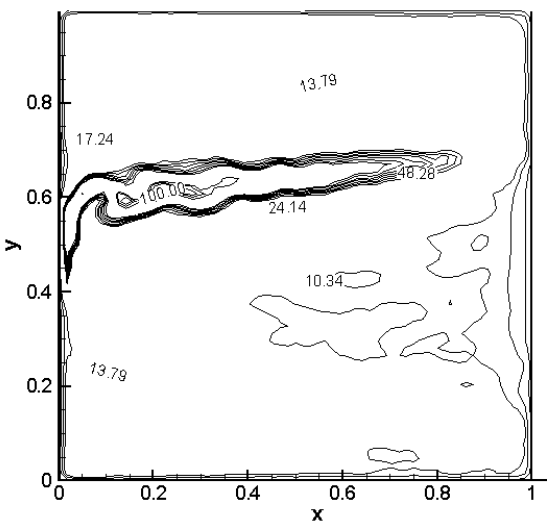

(b)
Arakawa. 1025

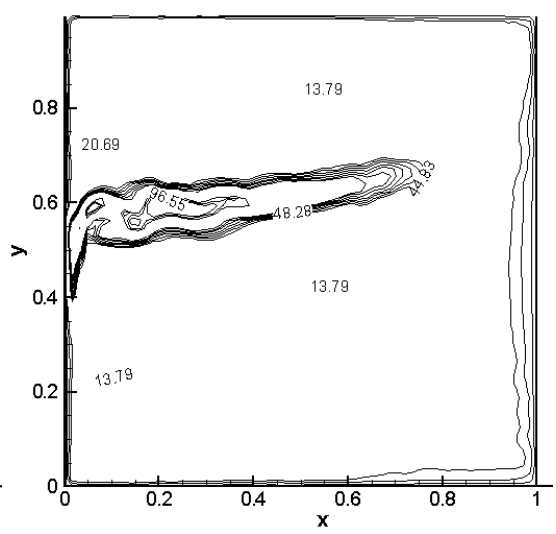

CABARET, 1025

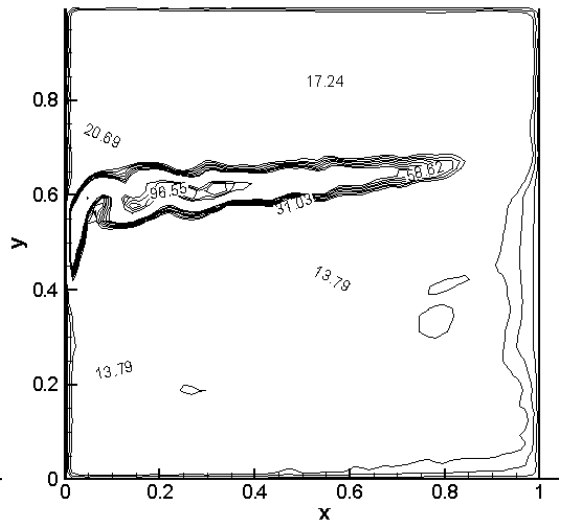

(c)

Figure 9. Convergence of the Arakawa and CABARET methods for the moderate-Re case. R.m.s. fluctuation of upper-ocean velocity streamfunction solutions: (a) 257 x 257 grid, (b) 513 x 513 grid, and (c) 1025 x 1025 grid. 
Arakawa, 257

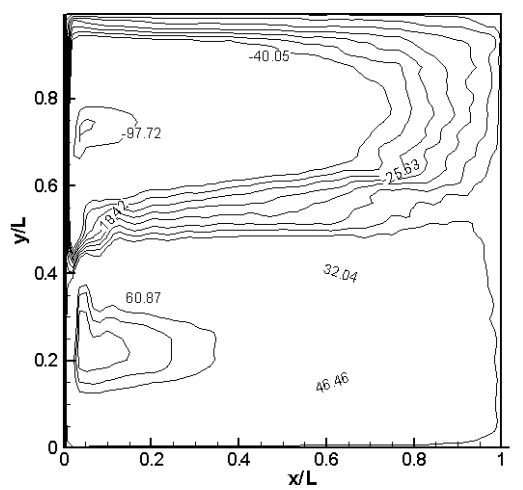

(a)

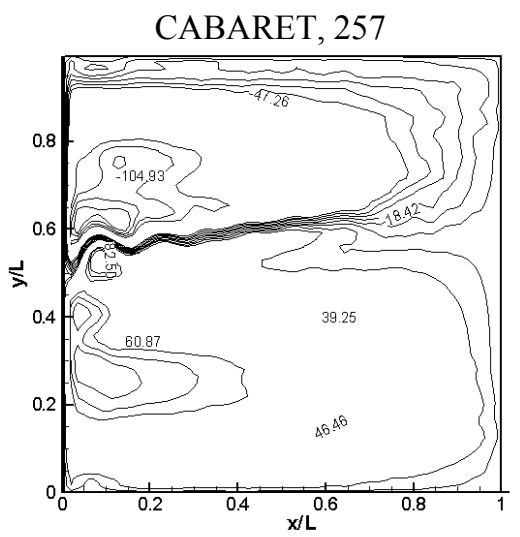

(b)

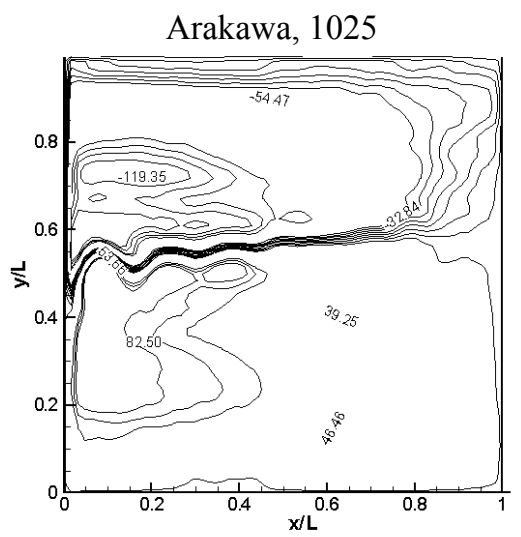

(c)

Figure 10. Comparative behaviour of solutions for the high-Re case. Time-averaged, upper-ocean velocity streamfunction solutions: (a) Arakawa method on 257 x 257 grid, (b) CABARET method on 257 x 257 grid, and (c) Arakawa method on 1025 x 1025 grid. 


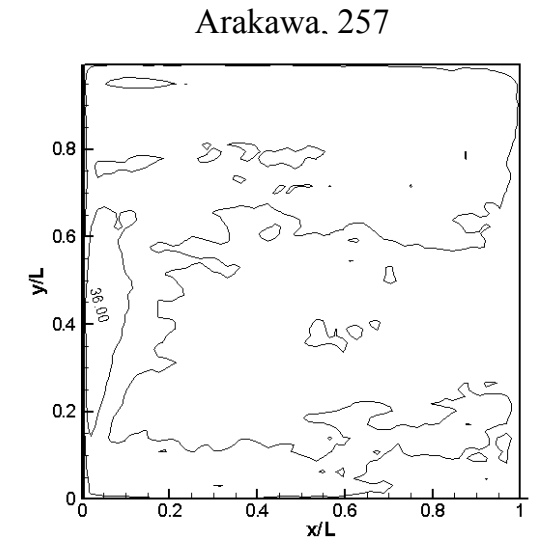

(a)

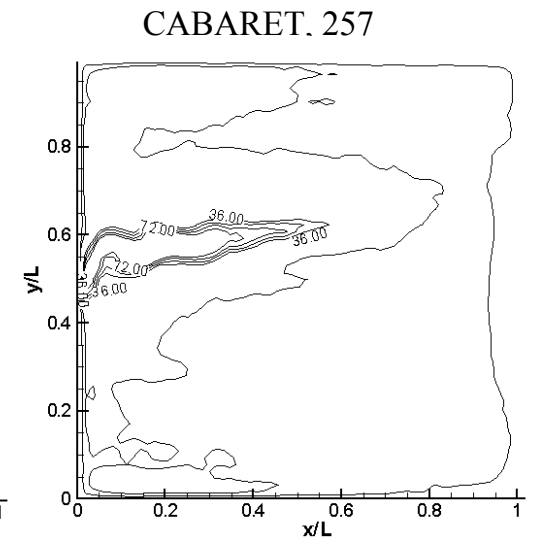

(b)

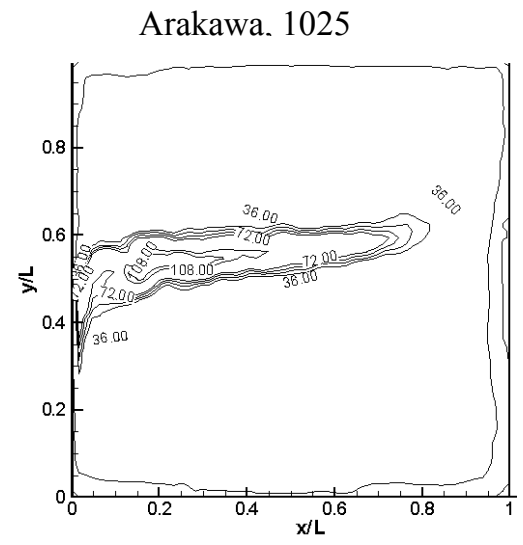

(c)

Figure 11. Comparative behaviour of solutions for the high-Re case. R.m.s. fluctuation of upperocean velocity streamfunction solutions: (a) Arakawa method on 257 x 257 grid, (b) CABARET method on 257 x 257 grid, and (c) Arakawa method on 1025 x 1025 grid. 


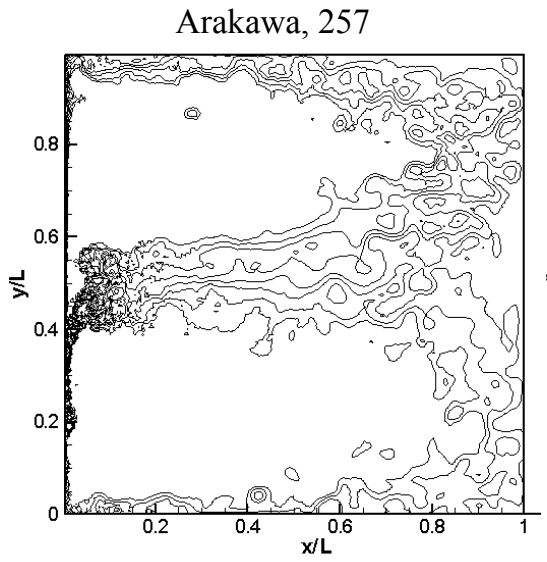

(a)

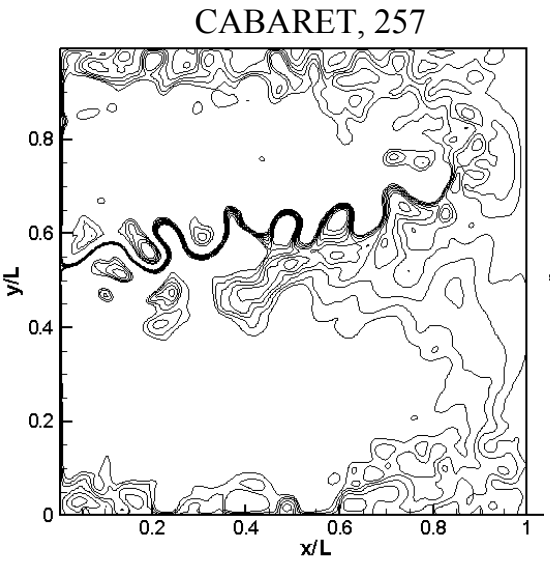

(b)

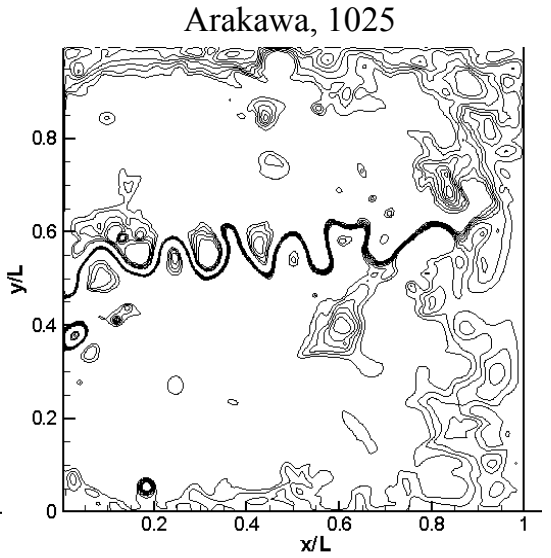

(c)

Figure 12. Comparative behaviour of solutions for the high-Re case. Typical instantaneous upperocean velocity streamfunction: (a) Arakawa method on 257 x 257 grid, (b) CABARET method on 257 x 257 grid, and (c) Arakawa method on 1025 x 1025 grid. 


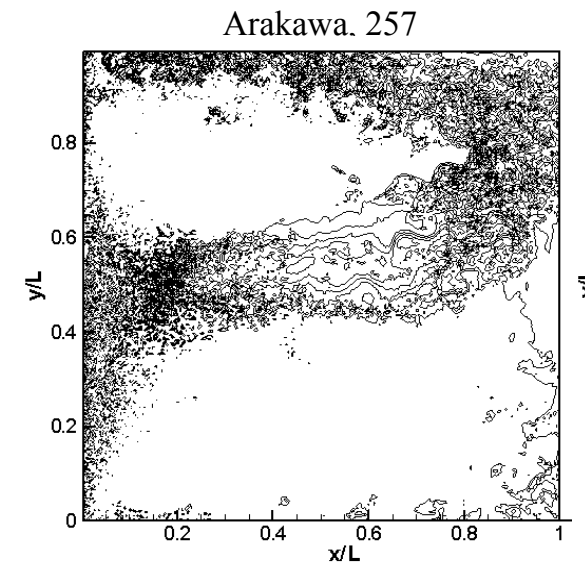

(a)

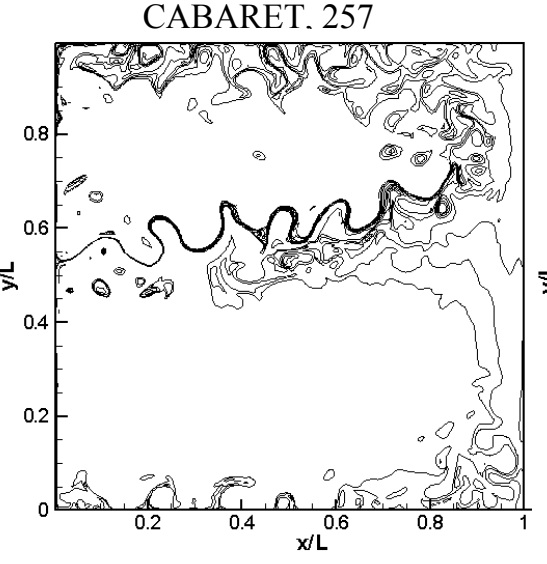

(b)

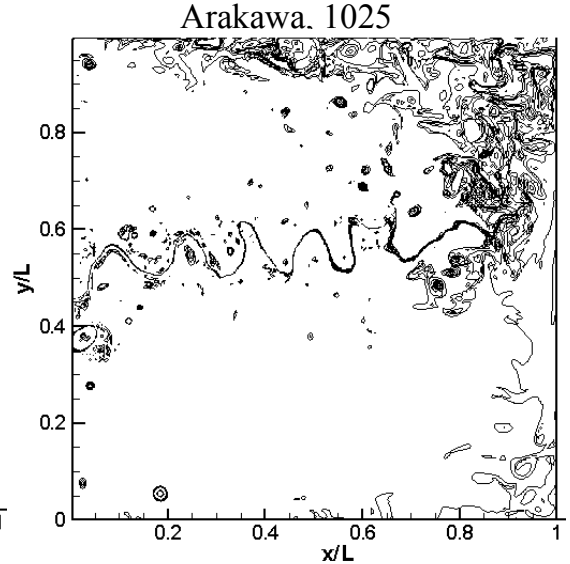

(c)

Figure 13. Comparative behaviour of solutions for the high-Re case. Typical instantaneous upperocean PV anomaly: (a) Arakawa method on 257 x 257 grid, (b) CABARET method on 257 x 257 grid, and (c) Arakawa method on 1025 x 1025 grid. 